\title{
The role of stellar feedback in the formation of galaxies.
}

\author{
Daniel Ceverino and Anatoly Klypin \\ Astronomy Department, New Mexico State University, Las Cruces, NM
}

\begin{abstract}
Although supernova explosions and stellar winds happen at very small scales, they affect the interstellar medium (ISM) at galactic scales and regulate the formation of a whole galaxy. Previous attempts of mimicking these effects in simulations of galaxy formation use very simplified assumptions. We develop a much more realistic prescription for modeling the feedback, which minimizes any ad hoc sub-grid physics. We start with developing high resolution models of the ISM and formulate the conditions required for its realistic functionality: formation of multi-phase medium with hot chimneys, super-bubbles, cold molecular phase, and very slow consumption of gas. We find that this can be achieved only by doing what the real Universe does: formation of dense $\left(>10 \mathrm{H}\right.$ atoms $\left.\mathrm{cm}^{-3}\right)$, cold $(T \approx 100 \mathrm{~K})$ molecular phase, where the star formation happens, and which is disrupted by young stars. Another important ingredient is the runaway stars: massive binary stars ejected from molecular clouds when one of the companions becomes a supernova. Those stars can move to 10-100 parsecs away from molecular clouds before exploding themselves as supernovae. This greatly facilitates the feedback. Once those effects are implemented into cosmological simulations, galaxy formation proceeds more realistically. For example, we do not have the overcooling problem. The angular momentum problem (resulting in a too massive bulge) is also reduced substantially: the rotation curves are nearly flat. The galaxy formation also becomes more violent. Just as often observed in QSO absorption lines, there are substantial outflows from forming and active galaxies. At high redshifts we routinely find gas with few hundred $\mathrm{km} \mathrm{s}^{-1}$ and occasionally $1000-2000 \mathrm{~km} \mathrm{~s}^{-1}$. The gas has high metallicity, which may exceed the solar metallicity. The temperature of the gas in the outflows and in chimneys can be very high: $T=10^{7}-10^{8} \mathrm{~K}$. The density profile of dark matter is still consistent with a cuspy profile. The simulations reproduce this picture only if the resolution is very high: better than $50 \mathrm{pc}$, which is 10 times better than the typical resolution in previous cosmological simulations. Our simulations of galaxy formation reach the resolution of $35 \mathrm{pc}$.
\end{abstract}

Subject headings: hydrodynamics, methods: n-body simulations, ISM: general, stars: formation, galaxies: formation, evolution

\section{Introduction}

The current cosmological paradigm, the $\Lambda \mathrm{CDM}$ Universe, has successfully explained the overall assembly of cosmic structures (Blumenthal et al. 1984: Davis et al. 1985; Spergel et al. 2007). In this picture ordinary matter ("baryons"), which emits and absorbs light, passively follows the evolution of the dark matter. This should be corrected, if we want to make a realistic theory of galaxy formation. It is necessary to include the physics of the gas and galaxy formation into the
$\Lambda \mathrm{CDM}$ paradigm, because, after all, many observational evidences of cosmic structures come from the light emitted by galaxies.

Galaxy formation is driven by a complex set of physical processes with very different spatial scales. Radiative cooling, star formation and supernova explosions happen at scales less than $1 \mathrm{pc}$, but they affect the formation of a whole galaxy (Dekel \& Silk 1986). In addition, large-scale cosmological processes, such as gas accretion through cosmic filaments, and galaxy mergers, control the galaxy assembly. As a result, a complex interplay 
between very different processes drives the formation of galaxies. Cosmological gasdynamical simulations have become useful tools to study galaxy formation.

In early cosmological simulations "galaxies" formed with too small disks and a significant fraction of angular momentum was lost Navarro \& Steinmetz 2000). The situation has improved in the last years. Sommer-Larsen et al. (2003); Governato et al. (2004); Kaufmann et al. (2006) show that a sub$\mathrm{kpc}$ resolution is necessary to prevent an artificial loss of angular momentum. Recent improvements in both the resolution and modeling of the feedback have resulted in simulations with extended galactic disks (Governato et al. 2004; Robertson et al. 2004; Brook et al. 2004; Okamoto et al. 2005; D'Onghia et al. 2006; Governato et al. 2007). However, simulated galaxies are still too concentrated, and more realistic simulations with better resolution and better physics are needed to reproduce the shape of the rotation curves of observed galaxies.

Current simulations lack the necessary resolution to follow correctly the effect of supernova explosions in the ISM. Because of this lack of resolution, the modeling of stellar feedback has relied on ad-hoc assumptions about the effect of stellar feedback at scales unresolved by simulations $(0.2-1 \mathrm{Kpc})$. Early attempts to introduce stellar feedback into simulations found the obstacle of a strong radiative cooling. The energy deposited by supernova explosions was quickly radiated away without any effect in the ISM (Katz 1992). Several shortcuts have been proposed to get around this over-cooling problem.

The most common method is to artificially stop cooling when the stellar energy is deposited Gerritsen \& Icke 1997; Thacker \& Couchman 2000; Sommer-Larsen et al. 2003; Kereš et al. 2005; Governato et al. 2007) This approach prolongs the adiabatic phase of supernova explosion (the Sedov solution) to about $30 \mathrm{Myr}$. The motivation behind this ad-hoc assumption is that the combination of blastwaves from different supernova explosions and turbulent motions produces hot bubbles much larger than individual supernova remnants and last longer. All this effects are not resolved with the current resolution. They do not develop in a self-consistent way. Instead, the delay in the cooling is introduced by hand.
The problem is that other effects can be missed at the same time due to a lack of resolution and an inaccurate modeling of feedback.

Another method is to introduce a sub-resolution model in which the energy from supernova explosions is stored in an unresolved hot phase, which does not cool and looses energy through the evaporation of cold clouds (Yepes et al. 1997; Springel \& Hernquist 2003). In this model, the only effect of stellar feedback is to regulate the star formation: the hot gas is coupled with the cold phase through cloud formation and evaporation. As a result, this high entropy gas is artificially trapped within the galactic disk. Thus, galactic winds are introduced in a simplified way in order to reproduce other natural effects of stellar feedback, such as galactic outflows.

An alternative approach assumes kinetic feedback instead of thermal feedback (Navarro \& White 1993). In that case, the energy from supernova explosions or stellar winds is transfered to the kinetic energy of the surrounding medium. This energy is not dissipated directly by radiative cooling. However, in order to resolve this effect accurately, simulations should be able to resolve the expansion of individual supernova explosions or the stellar winds from individual stars. Currently, this is not possible. At larger scales, the picture is more complicated. Different blastwaves from different supernova explosions can collide, dissipating their kinetic energy. The same dissipation of energy happens in collisions of stellar winds in stellar clusters. So, it is commonly assumed that most of the kinetic energy from stellar feedback is dissipated into thermal energy at the smallest scales resolved by simulations. Nevertheless, this feedback-heated gas can expand. As a result, thermal energy can be transfered to kinetic energy. The net results are flows at large scales powered by the thermal feedback. However, feedback heating should dominates over radiative cooling: only in this case those flows are produced.

To summarize, the main problems of current simulations of galaxy formation are the lack of the necessary resolution and too simplified models of the complex hydrodynamic processes in the multiphase ISM.

The galactic ISM has a very wide range of densities and temperatures (for review see Cox (2005) and Ferrière (2001)). Three distinct phases are 
distinguished: the dense cold gas (giant molecular clouds (GMC), cold HI gas or diffuse clouds) with densities above $10 \mathrm{~cm}^{-3}$ and temperatures bellow $100 \mathrm{~K}$, the warm component with densities between 0.1 and $1 \mathrm{~cm}^{-3}$ and temperatures of several thousands degrees, and the hot phase with temperatures above $10^{5} \mathrm{~K}$ and densities bellow $10^{-2} \mathrm{~cm}^{-3}$. This multiphase medium is set by the competition of cooling and heating mechanism and the onset of thermal instabilities. The hot ISM component $\left(T>10^{5} \mathrm{~K}\right)$ is usually associated with gas heated by shocks. They can be produced by turbulent motions driven by gravitational and thermal instabilities. However, these turbulent driven shocks can only heat the gas up to $10^{6} \mathrm{~K}$ (Wada \& Norman 2001). Only supernova explosions and stellar winds can produce larger gas temperatures (McCrav \& Snow 1979; Spitzer 1990).

2D and 3D hydrodynamical simulations of the ISM have enough resolution (parsecs) to resolve the multi-phase nature of the ISM and to explore complicated effects of stellar feedback on different scales (Rosen \& Bregman 1995; Scalo et al. 1998; Korpi et al. 1999; de Avillez 2000; de Avillez \& Breitschwerdt 2004, 2007; Wada \& Norman 2001, 2007; Slyz et al. 2005). There is much to learn from these simulations. However, they typically focus on conditions in the solar neighborhood, which are different from what one may expect during early stages of galaxy formation. Not always they follow the whole gas cycle: cooling, star formation, and stellar feedback. For example, de Avillez \& Breitschwerdt (2004) include star formation but artificially restrict the rate of supernova explosions around a fixed value. However, this rate could be much higher in large star forming regions. As a result, the effect of stellar feedback is underestimated in these regions. Nevertheless, the effect of the stellar feedback in the ISM, such as the formation of hot bubbles and super-bubbles is resolved.

It is crucial to understand where and how the energy from massive stars is released back to the ISM. While a large fraction of massive stars are found in stellar clusters and $\mathrm{OB}$ associations, $10-30 \%$ are found in the field, away from any molecular cloud or stellar cluster (Gies 1987; Stone 1991). This population have peculiar kinematics. Their velocity dispersion is about
$30 \mathrm{~km} \mathrm{~s}^{-1}$, much higher than the velocity dispersion of the population of massive stars in clusters $\left(10 \mathrm{~km} \mathrm{~s}^{-1}\right)$ (Stone 1991). Some of these stars have large peculiar velocities, up to $200 \mathrm{~km} \mathrm{~s}^{-1}$ (Hoogerwerf et al. 2000). This is why they are called runaway stars. The current scenario of the origin of runaway stars is the ejection of these massive stars from stellar clusters. There are two possible mechanisms of this ejection. One possibility is the ejection due to a supernova explosion in a close binary system (Zwicky 1957; Blaauw 1961). The second mechanism is the ejection due to dynamical encounters in the crowded regions of stellar clusters (Poveda et al. 1967). In spite of the fact that a significant fraction of the stellar feedback occurs far from star forming regions, no attention has been paid to its effect on the galaxy formation.

We first study the effect of stellar feedback in the ISM, using simulations of a Kpc-scale piece of the ISM with few parsecs resolution. Then, we check if this picture holds when the resolution is degraded to the resolution that our cosmological simulations can achieve at high redshift. Finally, we study the effect of stellar feedback in galaxy formation at high redshift using cosmological hydrodynamical simulations.

This paper is organized as follows. Section 2 describes the necessary conditions in which stellar feedback dominates over radiative cooling. Section 3 describes all details of the modeling of stellar feedback. Section 4 shows Kpc-scale simulations of the ISM. Section 5 describes the cosmological simulations of galaxy formation. Finally, section 6 is the discussion and conclusion. Throughout the paper we give quantities in physical units.

\section{Physical conditions for the heating regime}

The thermodynamical state of the gas depends on two competing processes: heating from stellar feedback and cooling from radiative processes. They appear as source and sink terms of internal energy in the equation of the first law of thermodynamics:

$$
\frac{d u}{d t}+p \nabla \cdot \mathbf{v}=\Gamma-\Lambda
$$

where $u$ is the internal energy per unit volume, $p$ is the pressure of the gas, and $\mathbf{v}$ is its velocity. 
Parameter $\Gamma$ is the heating rate due to stellar feedback, and $\Lambda$ is the net cooling rate from radiative processes.

The heating rate from stellar feedback can be expressed as the rate of energy losses from a young and active single stellar population with a given density, $\rho_{*, \text { young }}$ :

$$
\Gamma=\rho_{*, \text { young }} \Gamma^{\prime}
$$

where $\Gamma^{\prime}$ is the specific rate of energy losses of the stellar population according to its age. The cooling rate can be expressed as:

$$
\Lambda=n_{H}^{2} \Lambda^{\prime}
$$

where $n_{H}$ is the hydrogen number density.

\subsection{Heating versus radiative cooling}

Now, we can ask ourselves under which conditions the feedback heating dominates over the radiative looses. Using the expression, $n_{H}=$ $\rho_{\text {gas }} /\left(\mu_{H} m_{H}\right)$, where $\rho_{\text {gas }}$ is the gas density, $\mu_{H}$ is the molecular weight per hydrogen atom and $m_{H}$ is the hydrogen mass, the condition for heating $(\Lambda \leq \Gamma)$ can be expressed as:

$$
n_{H} \Lambda^{\prime} \leq \frac{\rho_{*, \text { young }}}{\rho_{\text {gas }}} \mu_{H} m_{H} \Gamma^{\prime}
$$

Using typical values, we can rewrite the condition for the heating regime in the following way:

$$
\begin{gathered}
\left(\frac{n_{H}}{0.1 \mathrm{~cm}^{-3}}\right)\left(\frac{\Lambda^{\prime}}{10^{-22} \operatorname{erg~s}^{-1} \mathrm{~cm}^{-3}}\right) \leq \\
\left(\frac{\rho_{*, \text { young }}}{\rho_{\text {gas }}}\right)\left(\frac{\Gamma^{\prime}}{10^{34} \mathrm{erg} \mathrm{s}^{-1} \mathrm{M}_{\odot}^{-1}}\right)
\end{gathered}
$$

The cooling rate, $\Lambda^{\prime}$, is a strong function of gas temperature. So, the temperature and the density of the gas are two key properties in establishing the cooling or the heating regimes. The following two examples illustrate common situations.

At temperatures around $10^{4} \mathrm{~K}$, the cooling rate is close to its maximum value. We use $\Lambda^{\prime}=10^{-22}$ erg $\mathrm{s}^{-1} \mathrm{~cm}^{3}$ as a fiducial value. In this case eq.(5) shows that the heating overcomes the cooling only at very low densities $n_{H} \leq 0.1 \mathrm{~cm}^{-3}$, optimistically assuming that the ratio of densities, $\rho_{*, \text { young }} / \rho_{\text {gas }}$ is about unity. As a result, stellar feedback is not able to heat the gas beyond $10^{4}$ $\mathrm{K}$ for densities higher than $0.1 \mathrm{~cm}^{-3}$ and typical values of $\Gamma^{\prime}$. This is the well known overcooling problem for simulations, which allow cooling only to a temperature of $10^{4} \mathrm{~K}$ at which the star formation is assumed to happen. The energy from stellar feedback is radiated away very efficiently and the thermal feedback cannot play any role. In this case one needs to invoke "subgrid physics" a guess how the system should react to the energy released by the stars.

The situation is completely different if the gas is allowed to cool to $100 \mathrm{~K}$. The cooling is very inefficient at that temperature: $\Lambda^{\prime}=10^{-25} \mathrm{erg}$ $\mathrm{s}^{-1} \mathrm{~cm}^{3}$. So, stellar feedback can produce the net gas heating even if the density is large: $n_{H} \approx 100$ $\mathrm{cm}^{-3}$ for $\rho_{*, \text { young }} \approx \rho_{\text {gas }}$. Our conclusion is that simulations should include cooling process bellow $10^{4} \mathrm{~K}$. The cold phase should be resolved in order to get a high efficiency of stellar feedback.

However, heating to high temperatures is still problematic because as the gas is heated, the cooling rate increases. So, the peak of the cooling rate at $10^{4} \mathrm{~K}$ is a bottle-neck for heating gas to higher temperatures. Nevertheless, temperatures of diffuse gas as high as $10^{6}-10^{7} \mathrm{~K}$ have been observed around star-forming regions such as the Rosette nebulae Townsley et al. 2003; Wang et al. 2007), M17 (Townsley et al. 2003), and the Orion nebula (Feigelson et al. 2005; Guedel et al. 2007). The main question is how young and massive stars can heat their surrounding medium to these high temperatures, if the original medium, in which they were born had high densities.

The answer likely depends on the distance from those young stellar clusters. At small 1-2 pc distances it is likely to have the collisions of stellar winds (Townsley et al. 2003; Feigelson et al. 2005) . At larger distances the heating is related with the formation of superbubbles: the cumulative effect of winds and shocks generated by many young stars. One way or another, the density of gas around the young stellar population decreases and the ratio $\rho_{*, \text { young }} / \rho_{\text {gas }}$ increases as the overpressured bubble of gas expands. Once the density goes below $0.1 \mathrm{~cm}^{-3}$, eq.(5) can be fulfilled even at $10^{4} \mathrm{~K}$. The net result is a heating regime, in which the surrounding gas can be heated to very high temperatures. In other words, the process 
starts with the expanding bubbles at low temperatures and then proceeds to a runaway overheating regime.

As an example, we consider a typical GMC with a mass of $10^{5} \mathrm{M}_{\odot}$ and a size of $50 \mathrm{pc}$. These are the typical values found in recent catalogs of GMCs in M33 (Rosolowsky et al. 2007), M31 and the Milky way (Sheth et al. 2008). Therefore, the mean density is $n_{H}=50 \mathrm{~cm}^{-3}$. This value seems low compared with typical observed densities of molecular clouds. However, GMCs are highly clumpy. High-density clumps are usually embedded in a low density inter-clump medium. As a result, the volume-averaged density inside clouds is much smaller than the typical observed mass-weighted density (McKee 1999).

Now, we consider an Orion-like stellar cluster formed at the center of the cloud. The mass of the stellar cluster is $5 \times 10^{3} \mathrm{M}_{\odot}$ (Hillenbrand \& Hartmann 1998). In a region of mass $10^{4} \mathrm{M}_{\odot}$, the stellar cluster has the ratio $\rho_{* \text {,young }} / \rho_{\text {gas }}$ equal to 0.5 , and the condition for heating, eq.(5), is fulfilled. This heating produces an over-pressured hot bubble with a pressure 100 times higher than the surrounding unperturbed medium. As a result, the bubble expands, the density decreases, and the ratio $\rho_{* \text {,young }} / \rho_{\text {gas }}$ increases. Then, we get a runaway bubble, which proceeds to blowing away all gas (Kroupa et al. 2001).

Simulations should resolve the expansion of bubbles over-pressured by stellar feedback. The density of young (and active) stars and the density of gas should be comparable at the smallest scales resolved by the simulations. The minimum value of the ratio $\rho_{* \text {,young }} / \rho_{\text {gas }}$ depends on the gas density (eq. 5). For moderate gas densities, $n_{H}=10-100 \mathrm{~cm}^{-3}$, the above ratio should be around 0.1-1.

The above condition can be achieved if the star formation efficiency, the fraction of the progenitor cloud consumed in stars is $10 \%-50 \%$ at the resolution scale. This high efficiency is consistent with the observed value of $10 \%-40 \%$ found in Galactic stellar clusters, Greene \& Young 1992; Elmegreen et al. 2000; Kroupa et al. 2001). Due to the fact that $80 \%$ of the Galactic star formation occurs in stellar clusters (Lada \& Lada 2003), this high efficiency of star formation should be considered in any star formation model which can resolve the sites where star formation occurs.

\subsection{Local gravity versus pressure gradient}

As we saw in the previous section, low densities are required in order to heat the gas beyond the peak of the cooling curve. Stellar feedback should evacuate the gas by creating an expanding bubble around young stellar clusters. However, the over-pressured bubble expands only if the pressure gradient overcomes self-gravity.

If we consider an over-pressured bubble of radius $R$ in a homogeneous medium of density $\rho$, we can derive a Jeans-instability type of condition. As a result, the bubble expands only if the difference in pressure with its surroundings, $\Delta P$, satisfies the following relationship:

$$
\Delta P / k \geq \frac{4 \pi}{3 k} G(\rho R)^{2}=10^{-1}\left(n_{H} R_{p c}\right)^{2}
$$

where $k$ is the Boltzmann constant, $G$ is the gravitational constant, and $R_{\mathrm{pc}}$ is the radius in pc. The above equation sets the conditions for the bubble expansion. For the Galactic plane the pressure is $P / k \sim 2 \times 10^{4} \mathrm{~cm}^{-3} K$ (Cox 2005). For example, a region of $50 \mathrm{pc}$ in radius and a density of $100 \mathrm{~cm}^{-3}$ will only expand, if the difference in pressure is bigger than $2 \times 10^{6} \mathrm{~cm}^{-3} \mathrm{~K}$. This can be achieved, if the bubble is over-pressured by more than 100 times. Stellar feedback can produce this overpressure just by raising the temperature from $100 \mathrm{~K}$ to $10^{4} \mathrm{~K}$. The resulted over-pressured region will expand, and the density as well as the cooling rate will decrease. So, the efficiency of stellar feedback increases, raising the temperature and pressure further.

Eq. 6] also sets a upper limit on the resolution. Using the equation of state of the ideal gas $P=$ $n k T$, where $n$ is the mean number density and $\mathrm{T}$ is the temperature of the gas, the over-pressured bubble should be resolved with a spatial resolution $X_{p c}=R_{p c} / 2$, such that the expansion is resolved:

$$
\left(\frac{X_{p c}}{75 \mathrm{pc}}\right)^{2} \leq\left(\frac{T}{10^{4} \mathrm{~K}}\right)\left(\frac{n_{H}}{10 \mathrm{~cm}^{-3}}\right)^{-1}
$$

As a result, for typical values of these overpressured regions, the resolution should be better than $\sim 70 \mathrm{pc}$. Otherwise, the bubble cannot overcome its self-gravity and cannot expand. 


\section{Stellar feedback model}

We assume a model of thermal feedback for the injection of energy from stellar winds and supernova explosions. The kinetic energy from these processes is efficiently dissipated into thermal energy due to shocks at scales bellow the spatial resolution.

The net thermal rate $(\Gamma-\Lambda)$ is used to update the internal energy in each step of the simulation. This approach is rather different than the deposition of energy. Instead, the energy injection from stellar feedback is treated in a self-consistent way along with the radiative looses.

\subsection{Heating rate from stellar feedback}

The heating rate from stellar feedback in a given volume element is modeled as the rate of energy losses from a set of single stellar populations present in that volume. This is just a generalization of eq.(2):

$$
\Gamma=\frac{1}{V} \sum_{i} M_{i} \Gamma^{\prime}\left(t_{i}\right)
$$

where $M_{i}$ and $t_{i}$ are the mass and the age of each single stellar population.

The modeling of the specific release of energy over time, $\Gamma^{\prime}$, is motivated by the results from population synthesis codes, such as STARBURST99 (Leitherer et al. 1999). Figure 11 shows different models of $\Gamma^{\prime}$ and the results of a STARBURST99 computation with a Miller-Scalo IMF from $0.1 \mathrm{M}_{\odot}$ to $100 \mathrm{M}_{\odot}$. Parameter $\Gamma^{\prime}$ is dominated by stellar winds from massive OB mainsequence stars and WR stars during the first few Myr. Later the energy is produced by corecollapse supernovae from stars more massive than $8 \mathrm{M}_{\odot}$. After $40 \mathrm{Myr}$, the release of energy comes from stellar winds of AGB stars and other less powerful sources, and the injection rate drops 6 orders of magnitudes. Supernovae Ia dominate the feedback at much longer time-scales. We assume a peak of the SNIa rate at 1 Gyr. However, this peak is 3 orders of magnitude lower than the contribution from core-collapse supernovae. This is because the energy from a population of SNIa is diluted over a much longer time scale than the energy from core-collapse supernovae.

We model $\Gamma^{\prime}$ with a constant rate of $1.18 \times 10^{34}$ $\operatorname{erg~s}^{-1} \mathrm{M}_{\odot}^{-1}$ over $40 \mathrm{Myr}$. This is equivalent to the

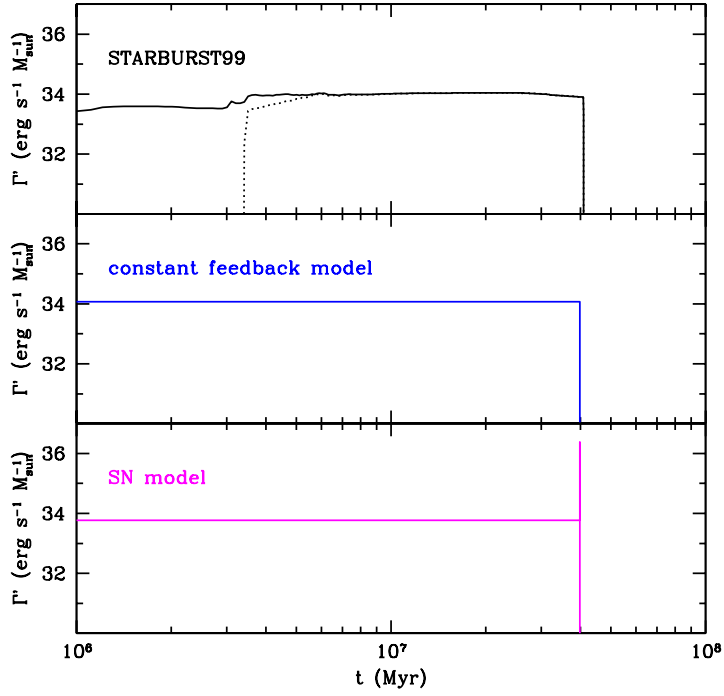

Fig. 1.- Rate of energy losses per unit mass from a single stellar population. Top panel shows the results from the STARBURST99 code, assuming a Miller-Scalo IMF for a mass range $(0.1-100) \mathrm{M}_{\odot}$. The dotted line shows the contribution of supernova explosions and the full line shows the total rate. Although supernova explosions dominate the overall energy release, stellar winds are the only mechanism of energy release during the first few Myr. Middle and bottom panels show two different models: a constant feedback model and a model of stellar wind plus core-collapse supernova. Although the total energy released is the same in both models, the SN model is more elementary and takes into account the explosive nature of core-collapse supernova.

injection of $2 \times 10^{51} \mathrm{erg}$ of energy from stellar winds and supernova explosions per each massive star with $M>8 \mathrm{M}_{\odot}$ during its lifetime. We assume a Miller-Scalo IMF in the mass range $(0.1-100) \mathrm{M}_{\odot}$. Note that this constant heating rate is the sum of the contributions from all massive stars in a single stellar population. We also consider a more simple model, which we call a SN model. In this case $10^{51}$ ergs is injected at constant rate due to stellar winds over 10 or $40 \mathrm{Myr}$. Then it follows a strong peak of energy release due to the supernova explosion, in which $10^{51} \mathrm{erg}$ are released during $10^{5} \mathrm{yrs}$ - the typical age of young supernova remnants. Although the total energy released is the same in 
both models, the SN model takes into account the explosive nature of core-collapse supernovae.

\subsection{A model of runaway stars}

The effect of runaway stars is implemented by adding a random velocity to a fraction of stellar particles (10\%-30\%). This extra velocity has a random orientation and the value is taken from an exponential distribution with a characteristic scale of $17 \mathrm{~km} \mathrm{~s}^{-1}$. This choice is motivated by Hipparcos data (Hoogerwerf et al. 2000) and Monte-Carlo simulations (Dray et al. 2005). For comparison, a Gaussian distribution is also used (Stone 1991). However, the effect of runaway stars in the ISM is not very sensitive to the details of this velocity distribution.

\subsection{Radiative cooling}

Radiative cooling counterbalances feedback heating. So it is very important to have an accurate model of radiative cooling in order to study the net effect of stellar feedback in the ISM.

We use the model of radiative cooling described in Kravtsov (2003). It is a metallicity-dependent cooling plus a UV heating due to a cosmological ionizing background (Haardt \& Madau 1996). The model includes Compton heating/cooling and molecular cooling. The temperature range of the model is between $10^{2} \mathrm{~K}<T<10^{9} \mathrm{~K}$. Thus, this model includes cooling below $10^{4} \mathrm{~K}$ and the gas can reach the thermodynamical conditions of molecular clouds. As we saw in section 2, this is crucial for the efficiency of the stellar feedback.

The cooling and heating rates from radiative processes are tabulated using the CLOUDY code (version 96b4; Ferland et al. 1998). As a result, the net cooling rate from radiative processes, $\Lambda^{\prime}$, is available for a given density, temperature, metallicity and redshift.

\subsection{Description of the code}

The numerical simulations were performed using the Eulerian gasdynamics + N-body Adaptive Refinement Tree code (Kravtsov et al. 1997; Kravtsov 1999, 2003). The physical processes of the gas include star formation, stellar feedback, metal enrichment, self-consistent advection of metals, cooling and heating rates from metallicity-dependent cooling and UV heating due to a cosmological ionizing background.

\section{Results of ISM runs}

Our first step in the understanding of stellar feedback in galaxies is to understand its effect in the ISM at galactic scales. Therefore, we run simulations of a $4 \times 4 \times 4 \mathrm{Kpc}^{3}$ piece of a galactic disk with 8-16 pc resolution. These simulations fully resolve the effect of massive stars at galactic scales. So, resolution is not longer an issue.

We can use this ISM-scale simulation as a benchmark for the effect of stellar feedback at galactic scales. Then, we can degrade the resolution to see which model of feedback reproduces the same overall picture at lower resolution. These simulations can then be used as testing grounds for these models at different resolutions. They tell us what are the necessary ingredients to reproduce the truly effect of stellar feedback at the resolution that we can afford in cosmological simulations of galaxy formation.

We want to see the effect of stellar feedback in the typical conditions of normal disk galaxies with moderate gas surface densities. So, we are not modeling starburst galaxies with large amounts of gas and high star formation rates. This type of study will be done in the future.

A $4 \mathrm{Kpc}$ box of ISM represents a significant piece of a galactic disk. The simulation resolves the dense galactic plane, where molecular clouds are formed. This is important to follow star formation correctly. At the same time, the simulation follows the gas at few Kpc above the galactic plane. This height is similar to the scale-height of the diffuse warm phase of the ISM (Cox 2005).

The simulation includes radiative cooling and UV heating from a uniform UV field at redshift 0 as described in section 3. Star formation happens in the highest density peaks with a density threshold of $100 \mathrm{~cm}^{-3}$. In each star formation event, $5 \%$ of the mass in gas inside a volume element is converted into a stellar particle with a mass of $88 \mathrm{M}_{\odot}$ within a time-step set by the Courant condition $\left(\sim 2 \times 10^{3}\right.$ yr $)$. The supernova model was used for stellar feedback and SNIa was not included. The metallicity was assumed solar and constant throughout the simulation. 


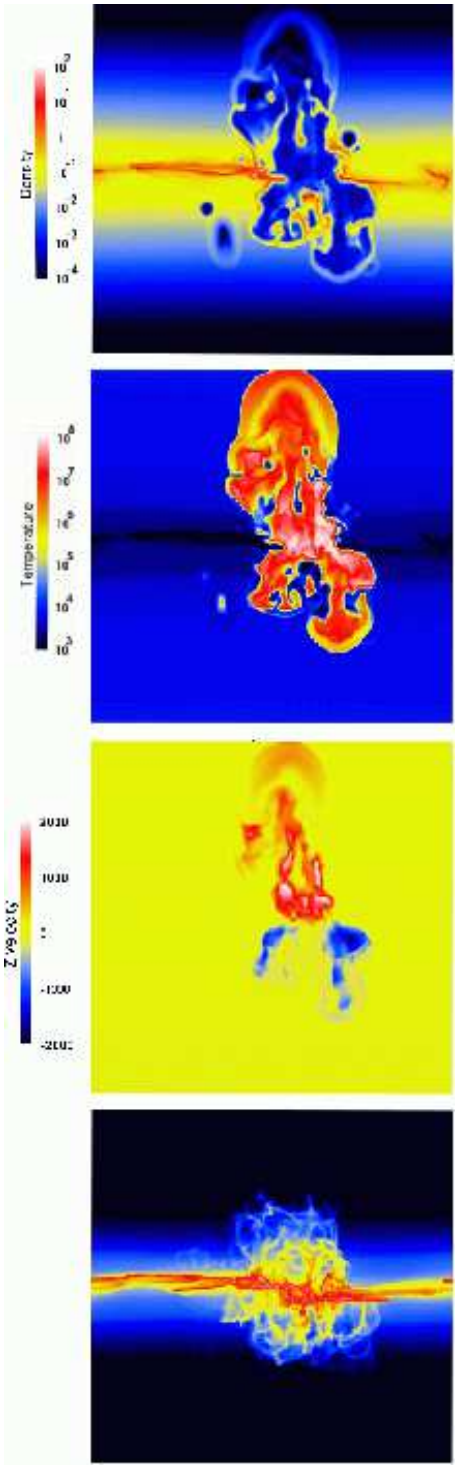

Fig. 2.- Formation of a galactic chimney. Edgeon slices through the simulation show density, temperature and velocity in the vertical direction, perpendicular to the galactic plane. The bottom panel shows gas column density. The chimney outflow is not a homogeneous, coherent flow: it is turbulent and has dense and cold clumps embedded into the flow. The core of the chimney reaches $10^{7}-10^{8} \mathrm{~K}$. Outflow velocities exceed $10^{3} \mathrm{~km} \mathrm{~s}^{-1}$. This hot material is able to escape the disk and generate a galactic wind.

\subsection{Initial conditions}

The initial distribution of gas density is uniform in the $x$ and $y$ directions of the box. In the $z$ direction, the density profile declines at both sides of the middle plane, $z=z_{0}=2 \mathrm{Kpc}$. This plane defines the galactic plane for this ISM model:

$$
n_{H}=n_{0} \cosh ^{-2}\left(\frac{z-z_{0}}{z_{d}}\right)
$$

where $n_{0}$ is the gas density in that plane and $z_{d}$ is the scale-height.

The choice of parameters sets the conditions of a quiescent normal galactic disk, $n_{0}=1 \mathrm{~cm}^{-3}$ and $z_{d}=250 \mathrm{pc}$. Thus, the surface density is ,$\Sigma_{\text {gas }}=16 \mathrm{M}_{\odot} \mathrm{pc}^{-2}$. The system is originally in hydrostatic equilibrium with a temperature of $10^{4} \mathrm{~K}$. No stars are present at the beginning of the simulation. The box has open boundaries in the z-direction. So, all material that cross these boundaries escapes the system.

The initial velocity field consists of a sum of plane-parallel velocity waves:

$$
\begin{aligned}
& u_{x}=\sum_{i, j, k} A_{x}(i, j, k) \sin (\vec{k} \cdot \vec{r}) \exp -\left(\frac{z-z_{0}}{z_{d}}\right)^{2} \\
& u_{y}=\sum_{i, j, k} A_{y}(i, j, k) \sin (\vec{k} \cdot \vec{r}) \exp -\left(\frac{z-z_{0}}{z_{d}}\right)^{2} \\
& u_{z}=\sum_{i, j, k} A_{z}(i, j, k) \sin (\vec{k} \cdot \vec{r}) \exp -\left(\frac{z-z_{0}}{z_{d}}\right)^{2}
\end{aligned}
$$

The amplitudes are taken from a Gaussian field with a tilted power spectrum, $P_{k} \propto k^{-3}$, where k is the wavenumber, $k=\frac{2 \pi}{L} \sqrt{i^{2}+j^{2}+k^{2}}$. i,j and $\mathrm{k}$ are integers running from -20 to 20 (excluding $0)$ and $u_{0}=20 \mathrm{~km} \mathrm{~s}^{-1}$. This is a typical spectrum of a compressible turbulent medium (Kraichnan 1967; Vázquez-Semadeni et al. 1995).

$$
\begin{aligned}
& A_{x}(i, j, k)=u_{0} \frac{R_{\text {Gauss }}}{\left(i^{2}+j^{2}+k^{2}\right)^{3 / 2}} \\
& A_{y}(i, j, k)=u_{0} \frac{R_{\text {Gauss }}}{\left(i^{2}+j^{2}+k^{2}\right)^{3 / 2}} \\
& A_{z}(i, j, k)=u_{0} \frac{R_{\text {Gauss }}}{\left(i^{2}+j^{2}+k^{2}\right)^{3 / 2}}
\end{aligned}
$$

$R_{\text {Gauss }}$ is a random number taken from a Gaussian distribution. 


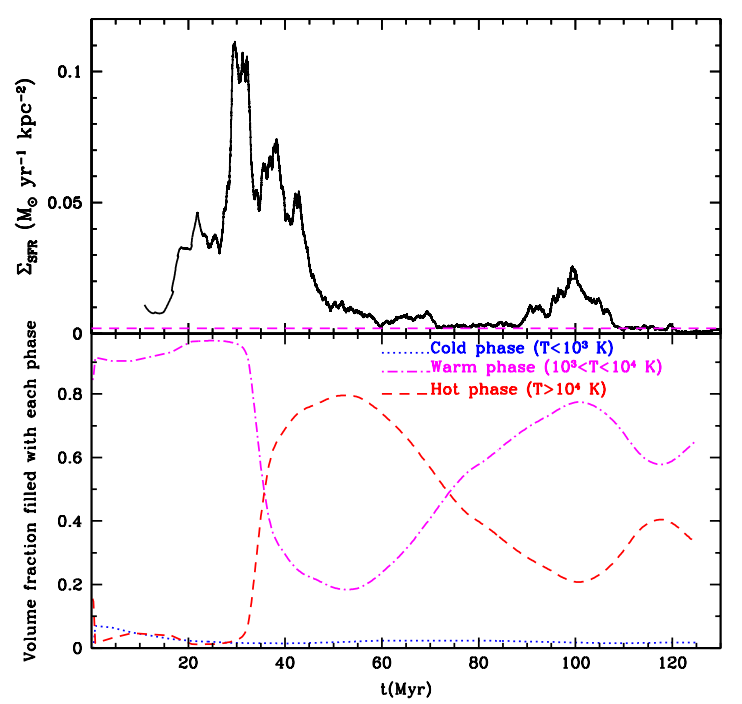

Fig. 3.- Top panel: Star formation rate surface density of the whole simulation. The value shown is also averaged over a period of $\sim 2 \times 10^{5} \mathrm{yr}$ (100 time-steps). After an initial burst, the star formation rate surface density is consistent with the Kennicutt et al. (2007) empirical fit (horizontal line). Bottom panel: Fraction of volume filled with each gas phase over time. The volume occupied by the warm and the hot phase oscillates. The hot phase dominates after a burst of star formation and the warm phase dominates when the gas is cooled down. The cold phase covers a small volume, which remains constant after an initial collapse.

\subsection{Galactic Chimney formation}

At the beginning of the simulation, the gas starts to move according to the turbulent velocity field. As a result, the gas accumulates where different flows converge and molecular clouds 1 naturally appear in form of filaments and shells. However, around $90 \%$ of the volume is filled with warm and diffuse gas heated by UV background. Star formation occurs in the cores of the cold phase. Newly formed massive stars inject energy and a cavity filled with hot and very diffuse gas is formed. This over-pressured material expands and the net result is the formation of super-bubbles.

\footnotetext{
${ }^{1}$ cold and dense phase with $n_{H} \geq 30 \mathrm{~cm}^{-3}$ and $T \leq 300 K$
}

This hot gas cannot stay in the plane of the disk, as a result, the bubble expands faster in the direction perpendicular to the disk, because the density declines in that direction. The bubble develops into a galactic chimney (Norman \& Ikeuchi 1989). The chimney outflow does not look as a homogeneous, coherent flow. Instead, the chimney is turbulent and has dense and cold clumps embedded into the flow. Eventually, the gas expands in the halo and cools (Figure 2).

Another interesting feature seen in this model is a population of isolated bubbles in the warm medium. These are the results of individual supernova explosions of runaway stars

\subsection{Star formation rate}

After an initial burst of star formation, the star formation rate is nearly constant for the rest of the evolution (Figure 3). We found a low star formation rate surface density, $\Sigma_{\mathrm{SFR}}=3 \times$ $10^{-3} \mathrm{M}_{\odot} \mathrm{yr}^{-1} \mathrm{Kpc}^{-2}$, temporally averaged over a period of $2 \times 10^{5} \mathrm{yr}$ (100 time-steps). This value is consistent with the expected value from the correlation between the star formation rate surface density and the gas surface density found in nearby galaxies Kennicutt 1998; Kennicutt et al. 2007). For a gas surface density of $\Sigma=12$ $\mathrm{M}_{\odot} \mathrm{pc}^{-2}$ at $\mathrm{t}=90 \mathrm{Myr}$, the expected value from the Kennicutt et al. (2007) fit is $\Sigma_{\mathrm{SFR}}=2 \times$ $10^{-3} \mathrm{M}_{\odot} \mathrm{yr}^{-1} \mathrm{Kpc}^{-2}$. This is very close to our results.

As observers usually do, we also calculate the gas consumption time-scale, $\tau=\mathrm{M}_{\mathrm{GMC}} / \mathrm{SFR}$, in the simulated molecular clouds, assuming that gas with a density higher than $30 \mathrm{~cm}^{-3}$ is mainly within GMCs. In our simulations, the amount of gas in molecular clouds is $\mathrm{M}_{\mathrm{GMC}}=8 \times 10^{6} \mathrm{M}_{\odot}$ at $\mathrm{t}=90 \mathrm{Myr}$. The star formation rate at that time is $\mathrm{SFR}=4.8 \times 10^{-2} \mathrm{M}_{\odot} \mathrm{yr}^{-1}$. As a result, the gas consumption time-scale in the simulated clouds is $\tau \approx 170 \mathrm{Myr}$. This is quite long compared with the typical free-fall time-scale inside molecular clouds, $\mathrm{t}_{\mathrm{ff}}=(3 \pi / 32 G \rho)^{1 / 2} \approx 4 \mathrm{Myr}$ for $n_{H}=100 \mathrm{~cm}^{-3}$.

In our simulations, the star formation efficiency over a free-fall time-scale, the fraction of gas consumed in stars during a free-fall time-scale, is only $2.5 \%$. This value is consistent with observations (Zuckerman \& Evans 1974). Krumholz \& Tan (2007) report a range of $0.6 \%-2.6 \%$ for the whole 


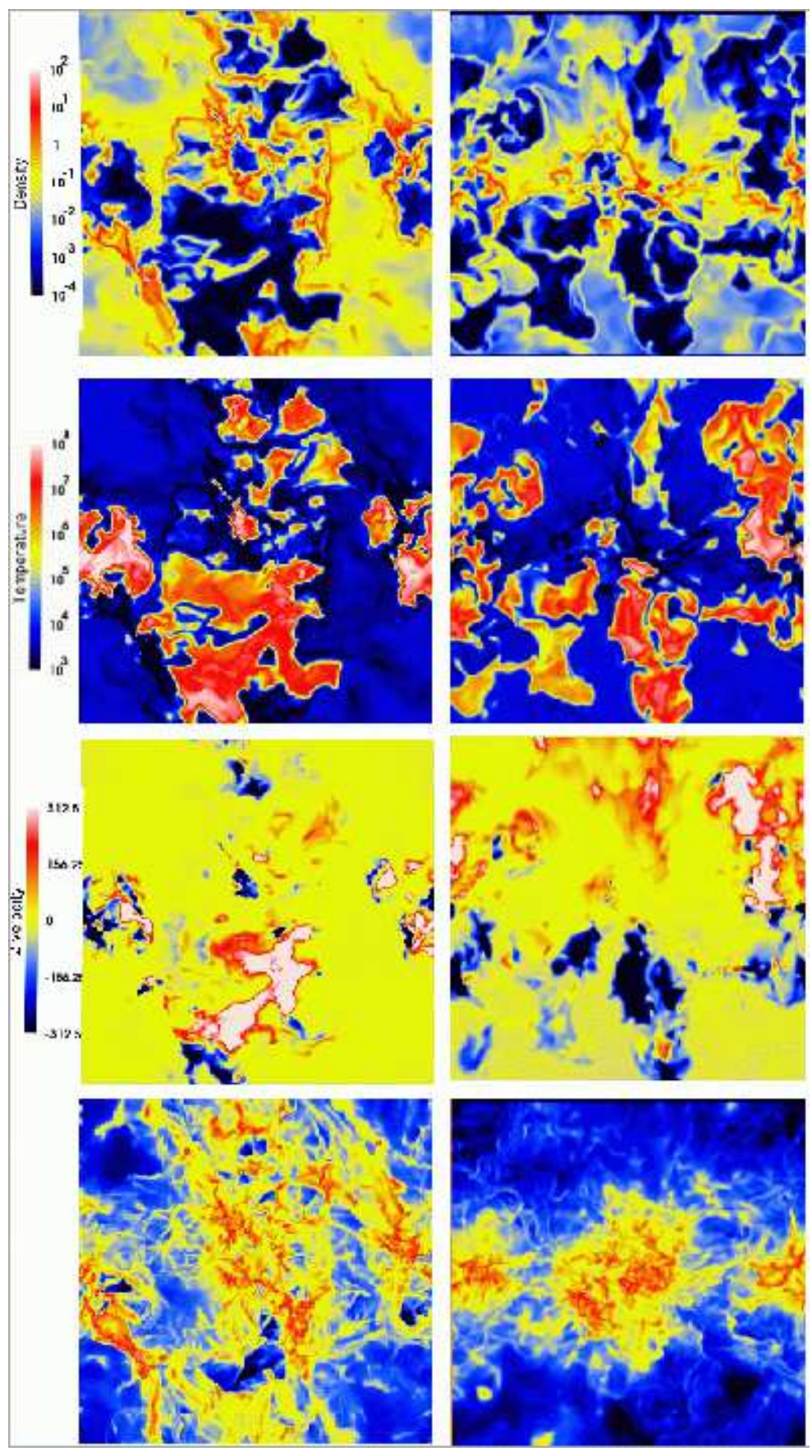

Fig. 4. - Snapshot of the model after $113 \mathrm{Myr}$, showing the density in $\mathrm{cm}^{-3}$ (first row), temperature in Kelvin (second row), gas velocity in the z-direction (third row), and surface density in $\mathrm{cm}^{-2}$ (forth row). Left panels show a face-on view of the galactic plane $\left(z=z_{0}\right)$ and right panels show an edge-on view perpendicular to that plane. The three phases of the ISM are clearly visible: cold and dense clouds, warm and diffuse medium and hot bubbles with very low densities. Velocities exceeding $300 \mathrm{~km} \mathrm{~s}^{-1}$ can be seen in hot outflows at both sides of the galactic plane. 
population of GMCs of the Milky-Way. Our value is also consistent with an efficiency of $\sim 3 \%$ found in simulations of GMCs (Vázquez-Semadeni et al. 2003; Clark et al. 2005). Finally, our results also agree with the model of a turbulent-dominated GMC, described in Krumholz \& McKee (2005). They give an efficiency per free-fall time-scale of $1.5 \%-3 \%$ for typical values of their model.

After $100 \mathrm{Myr}$, only $10 \%$ of the gas in the simulation has been converted into stars. Our simulations still have plenty of cold $\left(T \leq 10^{3} \mathrm{~K}\right)$ gas after $100 \mathrm{Myr}$. The surface density of this cold gas is $\sim 5 \mathrm{M}_{\odot} \mathrm{pc}^{-2}$. This value agrees with the surface density of molecular and atomic hydrogen of $\sim 6 \mathrm{M}_{\odot} \mathrm{pc}^{-2}$ found at the solar radius (Ferrière 2001). However, the surface density of molecular gas is low, $\sim 0.5 \mathrm{M}_{\odot} \mathrm{pc}^{-2}$, compared with the observed value of $\sim 2.5 \mathrm{M}_{\odot} \mathrm{pc}^{-2}$ (Ferrière 2001). This partially explains why our star formation efficiency over a free-fall time-scale is in the higher end of the observed range.

To conclude, stellar feedback is able to regulate star formation on galactic scales because it regulates the amount of gas available for star formation. Stellar feedback heats and disperses the cold and dense gas after a star formation event. In a single star formation event, a stellar particle of $\sim 90 \mathrm{M}_{\odot}$ is created. This roughly means the formation of a single high-mass star embedded in a small stellar cluster. Due to the resolution limit, our simulation can not follow the details of the star formation process bellow $\sim 10$ pc scales, only the overall net effect. This effect is the formation of a small stellar cluster with an efficiency of $5 \%$. As we pointed in $\S 2.1$, the star formation efficiency of Galactic stellar clusters is high, regardless the details of their formation. However, although the star formation efficiency is high, subsequent feedback processes produce a low average star formation.

\subsection{Volume filling factors in the ISM}

Figure 3 also shows that the net effect of stellar feedback is to produce the hot phase of the ISM. After the initial strong burst of star formation, This phase can cover up to $80 \%$ of the total volume. This represents almost the entire volume above a height of $400 \mathrm{pc}$ from the galactic plane. However, pockets of warm gas are embedded in this hot flow even at $2 \mathrm{Kpc}$ away from the plane.
It has the same inhomogeneous structure seen in the galactic fountain of figure 2. After $100 \mathrm{Myr}$, $\sim 25 \%$ of the gas is able to scape the computational volume.

Most of the hot gas is lost or cooled down after $100 \mathrm{Myr}$. As a result, the volume of hot gas decreases because the star formation is low and the injection of energy is lower than in the initial burst. The simulation settles into a more quiescent regime in which the volume occupied by the warm and hot phases oscillates in a self-regulated gas cycle. In this cycle, bursts of star formation (much smaller than the initial one) produce superbubbles and galactic chimneys of hot gas. Therefore, the volume of hot gas increases. As the star formation fades, the bubbles cool down and the fraction of hot gas decreases until the next stellar burst. This pattern reflects the star formation history. The particular fraction of hot and warm phases at any moment does depend to the particular star formation history of 10-40 Myr before that moment.

\subsection{Late stages of evolution}

The latter stages of the simulation offer a more representative view of the ISM. The effect of the initial conditions is gone. Therefore, we can study the characteristics of this feedback-driven ISM. We select a snapshot at $113 \mathrm{Myr}$, after the second burst of star formation. At that moment, the warm phase covers $\sim 60 \%$ of the volume and the hot phase filled $\sim 40 \%$. X-ray emitting gas with temperatures above $10^{5.5} \mathrm{~K}$ occupies $\sim 20 \%$ of the volume inside a height of $250 \mathrm{pc}$ above the galactic plane. This is roughly consistent with ISM simulations with a 1-pc resolution (de Avillez \& Breitschwerdt 2004), Galactic ISM models and observations (Ferrière 1998).

Figure 4 shows representative slices of the box. The medium is very inhomogeneous at different scales. Large bubbles of low density coexist with long filamentary structures of dense clouds. Overall, the medium covers more than 6 orders of magnitude in density and temperature. The cold phase forms dense and cold clouds near the galactic plane. The warm phase fills old cooled bubbles and low-density clouds. Finally, the hot phase is present in form of hot bubbles of few hundred pc wide and Kpc-scale chimneys. The gas in these chimneys is flowing away from the plane 


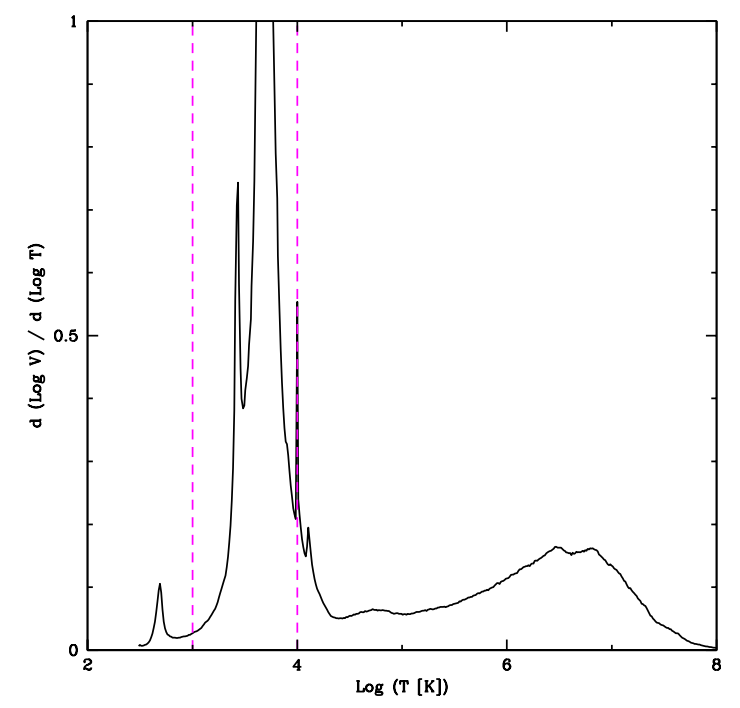

Fig. 5.- Distribution of the gas temperature at 113 Myr. The distribution has three different peaks corresponding to three different gas phases of the ISM: cold, warm and hot. Two vertical lines show the temperature cuts used throughout the paper at $T=10^{3} \mathrm{~K}$ and $T=10^{4} \mathrm{~K}$.

with velocities exceeding $\pm 300 \mathrm{~km} \mathrm{~s}^{-1}$. These bubbles even break the dense plane in hot spots surrounded by cold and dense shells. All this phenomenology associated with the hot phase is driven by stellar feedback. As a result, one effect of the stellar feedback is to sustain a three-phase ISM.

The distribution of temperature clearly shows the three main peaks of the three phases of the ISM (Figure 5). The two local minima correspond to thermally unstable gas. The minimum around $10^{3} \mathrm{~K}$, between the peaks of the cold and warm phases, is produced by the competition of UV heating and radiative cooling. This corresponds to the unstable regime of the classical two-phase model of the ISM in thermal equilibrium (Cox 2005). The dip between $10^{4}$ and $10^{5} \mathrm{~K}$ results from the peak of the cooling curve. The gas cools very fast at these temperatures. As a result, it usually appears at the interface between hot and warm gas. As an exception, old bubbles at these temperatures are present in the simulation with very low densities and far away from the plane.

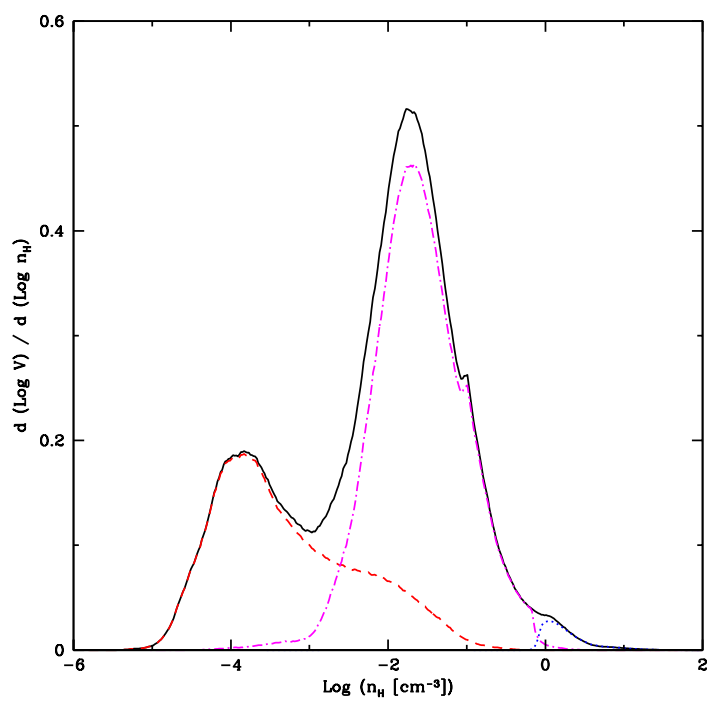

Fig. 6.- Density distribution at $113 \mathrm{Myr}$ and the contribution of the three phases. The dotted curve shows the cold phase $\left(T<10^{3} \mathrm{~K}\right)$, the dashed curve shows the hot phase $\left(T>10^{4} \mathrm{~K}\right)$, and the dash-dotted curve shows the warm phase $\left(10^{3} \mathrm{~K}<\right.$ $\left.T<10^{4} \mathrm{~K}\right)$. The distribution is clearly bimodal. The peaks correspond to the hot and warm phases. The cold phase dominates the high-density tail.

So, their cooling time is very long. This temperature distribution supports the temperature cuts used throughout the paper to distinguish the three phases: $10^{3} \mathrm{~K}$ for the cut between cold and warm phases and $10^{4}$ for the warm-hot cut. To summarize, this model of the ISM reproduces the main properties of the temperature distribution of the ISM (Cox 2005) and predicts that gas with very high temperatures $10^{7}-10^{8} \mathrm{~K}$ exists in the cores of galactic chimneys. This gas occupies only $5 \%$ of the total volume and have a very small surface density of $4 \times 10^{-6} \mathrm{M}_{\odot} \quad \mathrm{pc}^{-2}$.

These three phases of the ISM are also clearly visible in the density distribution (Figure 6). We use the temperature cuts defined before to see the contribution of the different phases. Thus, the hot phase dominates the low-density range, bellow $10^{-3} \mathrm{~cm}^{-3}$. The warm phase covers intermediate densities, and the cold phase dominates the high density tail above $1 \mathrm{~cm}^{-3}$. The density distribution of any of the three phases cannot be described 


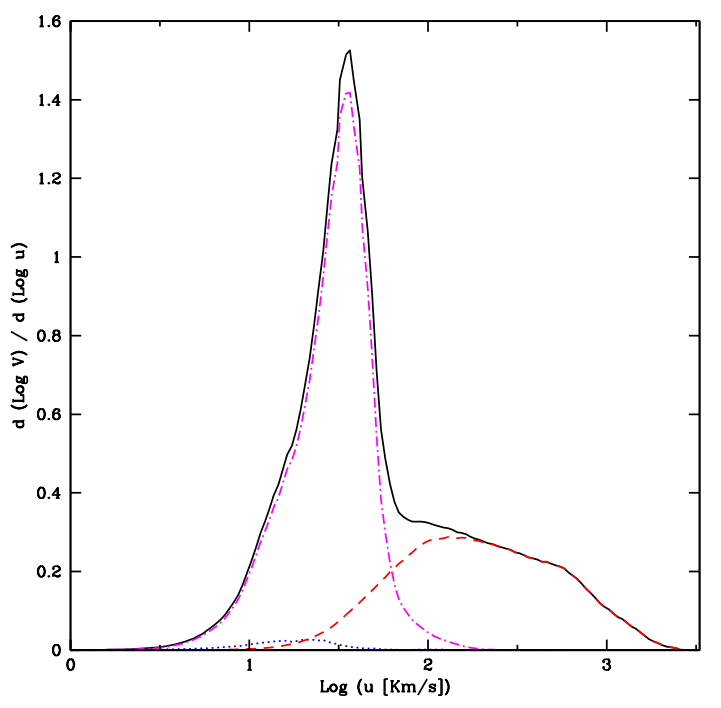

Fig. 7.- Distribution of gas velocity at $113 \mathrm{Myr}$. The curves represents the 3 gas phases as in figure 6. Cold and warm phases have moderate velocities, mostly bellow $100 \mathrm{~km} \mathrm{~s}^{-1}$. The hot phase dominates the high velocity tail of the distribution with velocities up to $2000 \mathrm{~km} \mathrm{~s}^{-1}$. These are outflows of gas which escapes the system.

by a single lognormal distribution, as claimed in Wada \& Norman (2001). Instead, a combination of several lognormal distributions may give a better approximation (Robertson \& Kravtsov 2007).

The distribution of velocities (Figure 7) shows two distinct features. The warm phase contribute to a strong peak around $30 \mathrm{~km} \mathrm{~s}^{-1}$. The hot phase dominates the high velocity tail. It has velocities as higher as $2000 \mathrm{~km} \mathrm{~s}^{-1}$. The gas with velocities in this tail can easily escape the system. This gas forms hot outflows and galactic chimneys.

Finally, figure 8 shows the distribution of the Mach number, $\mathrm{M}=\mathrm{u} / \mathrm{c}$, where $\mathrm{u}$ is the gas velocity and $\mathrm{c}$ is the sound speed. The distribution shows that $80 \%$ of the volume has supersonic motions. Almost all the warm phase, half of the hot phase and all the cold phase are supersonic flows. The subsonic range is dominated by the hot phase, In conclusion, the ISM can hold high supersonic motions, driven by stellar feedback.

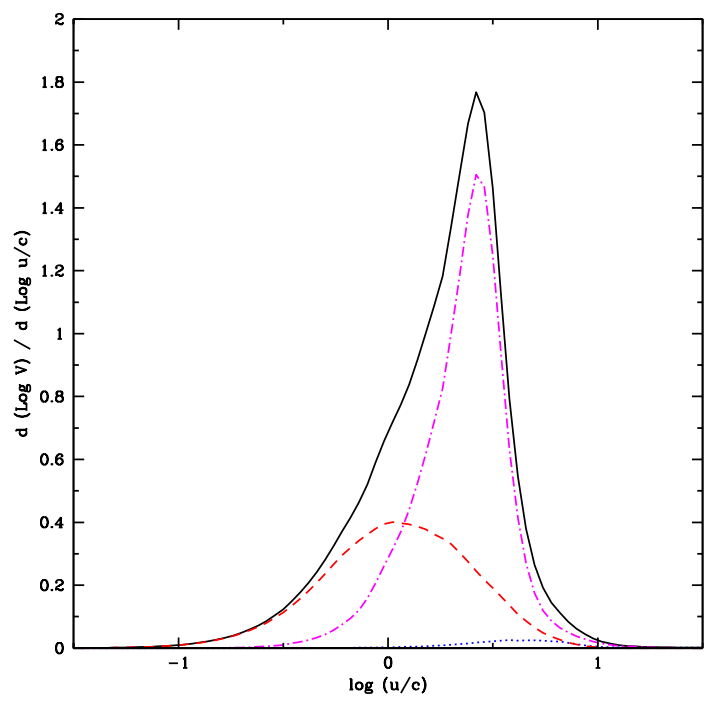

Fig. 8.- Distribution of the Mach number $(\mathrm{u} / \mathrm{c})$. The curves represents the 3 gas phases as in figure 6. $80 \%$ of the gas has supersonic motions. half of the hot phase has subsonic motions

\subsection{Degrading resolution}

The resolution in cosmological simulations of galaxy formation is much lower than the simulations of the ISM presented before. So, we can wonder if this picture of stellar feedback can hold if the resolution is degraded. Therefore, the same ISM models have been performed with high resolution $(14 \mathrm{pc})$ and with low resolution $(60 \mathrm{pc})$. The fraction of volume filled with each gas phase is used as a proxy to check the global effect of stellar feedback in the ISM (Figure 9). Left panels show that the hot phase covers a significant volume.

At low resolution and without runaway stars (top right panel), the hot gas is almost absent from the simulation. Small filaments are not resolved and the subsequent star formation is suppressed in these areas which can be easily broken by stellar feedback. As a result, star formation is concentrated at the center of big clumps of gas. Stars inject energy in high density regions, so this energy is radiated away without any thermodynamical effect in the medium.

However, if the model of runaway stars is included, the hot phase is recovered at low resolution. Stars can now migrate away from high den- 


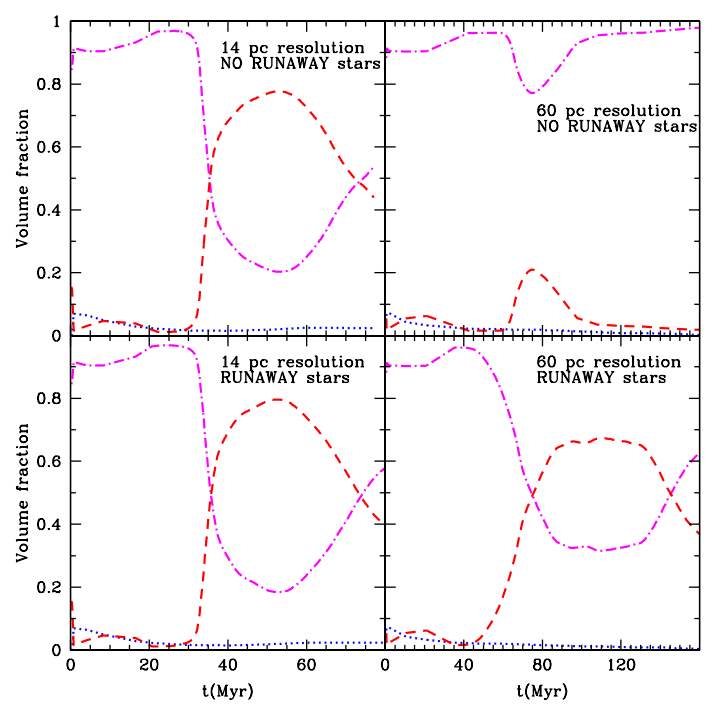

Fig. 9.- The panels show the evolution of the volume occupied by each phase of the gas in four different models. The curves represents the 3 gas phases as in figure 3. The hot phase is almost lost for the low resolution run without runaway stars (top right panel). If runaway stars are included, the hot phase is recovered at low resolution. As a result, a fraction of runaway stars produces an effect on the global ISM and its more evident in low resolution runs.

sity regions, so the injection of energy is more efficient in forming hot gas. As a result, the model with runaway stars can reproduce the effect of stellar feedback even at a resolution of $60 \mathrm{pc}$.

\subsection{The expansion of a hot bubble}

As a example of the conditions of the overheating regime discussed in section 2 , we can ask now how a hot bubble develops in the first place. The top panel of figure 10 shows the physical conditions of a single volume element over $10 \mathrm{Myr}$. This volume develops a hot and dilute medium starting from a cold and dense phase. The gradients are computed using a 3 -points finite differences expression using the adjacent cells.

At the beginning, there are no stars present inside that volume. So, there is no feedback heating. At the same time, the density is high enough so the the radiative cooling dominates over the UV back-
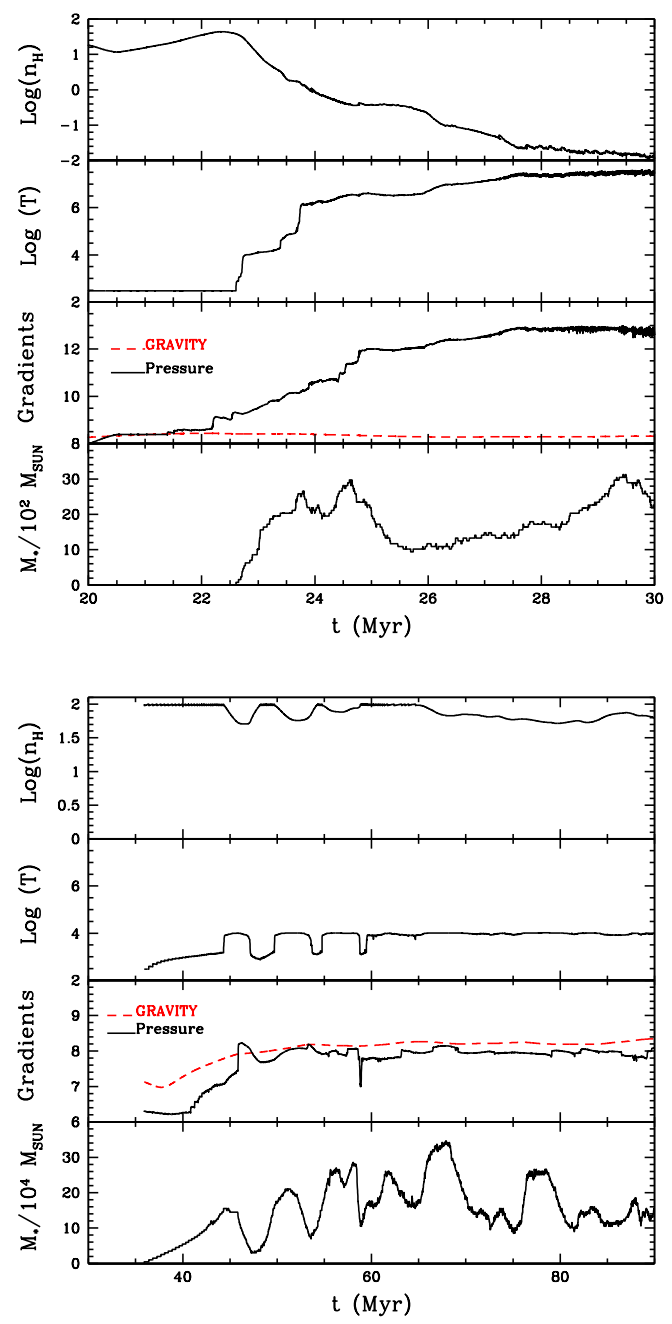

Fig. 10.- Evolution of the properties of a single volume element (Temperature, density, gradients of pressure and gravity, and the mass in young stars) in a run with 8 pc resolution (top panel) and with a resolution of $30 \mathrm{pc}$ (bottom panel). The top panel shows an over-pressured volume that expands due to stellar feedback. It produces a hot cavity filled with low-density gas. The bottom panel shows how this hot bubble cannot develop when the gradients of pressure do not dominate over gravity.

ground heating. As a result, the medium stays at the floor temperature of $300 \mathrm{~K}$. The medium is also in hydrostatic equilibrium.

The situation drastically changes when young 
stars appear. They are not born inside that particular volume. Instead, they are drifting slowly from adjacent cells. The result is that this young population injects energy into the medium. So, heating dominates over cooling initially. The system responds by increasing the temperature. As a result, the cooling rate increases and the medium reaches a balance between cooling and heating rates in a very short time-scale. This is because the cooling time is very short in those conditions. The net result is a medium slightly hotter than surroundings so this over-pressured region expands and the density inside that volume decreases.

Around $10^{4} \mathrm{~K}$, the cooling curve is a very steep function of temperature, so the temperature increases very slowly. But, at the same time, the density drops faster. As a result, the cooling rate decreases. This expansion is fueled by a roughly constant injection of energy from massive stars.

When the conditions of eq. 5 are fulfilled, the medium can pass through the peak of the cooling curve, somewhere between $10^{4}-10^{5} \mathrm{~K}$. After that, the gas has low density and a temperature of few millions degrees. As a result, heating dominates over cooling and a hot cavity is formed.

The bottom panel of figure 10 shows a different situation. The volume is selected to be the highdensity core of a molecular cloud formed in the low-resolution run shown in the top-right panel of figure 9. Stellar feedback from the stars formed in that core are able to heat the gas only to $10^{4} \mathrm{~K}$. The gradients of pressure do not overcome gravity. The condition of bubble expansion is not fulfilled , eq. (7). As a result, the density remains high and a hot bubble can not develop.

\section{Results of Cosmological runs}

In previous sections, we have shown that our models of stellar feedback follow the effect of supernova explosions and stellar winds in the ISM with a resolution of about $50 \mathrm{pc}$. The result is the formation of super-bubbles and galactic chimneys. Both are filled with hot and dilute gas. The net result is a multi-phase ISM and galactic outflows with large velocities.

Now, we can study the effect of stellar feedback in galaxy formation. We apply these feedback models in cosmological hydrodynamics simulations with a similar resolution of 35-70 pc. The
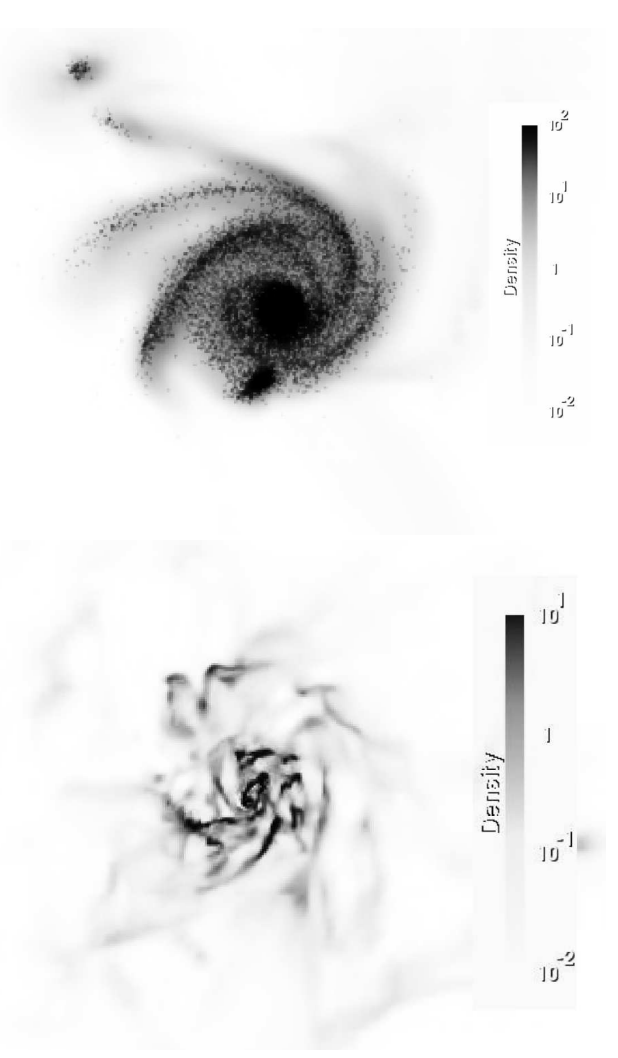

Fig. 11.- Density-weighted average gas density along the line-of-sight through a MW progenitor at redshift 3. The top panel shows a smooth gas distribution with some density enhancement due to a pattern of spiral arms. Young stars appear as points. They follow the spiral pattern. However, the distribution has a very concentrated and dense center. The bottom panel shows the heating regime case. The distribution is not as dense, so the range of density is different. The center is less concentrated and the distribution looks clumpy with dense filaments and clouds embedded in a more diffuse medium. This is the case of a multiphase ISM. The size of the images is $30 \mathrm{Kpc}$ in both cases.

simulations follows the formation of a MW-type galaxy starting from primordial density fluctuations.

The computational box is $10 h^{-1} \mathrm{Mpc}$ commoving box. We apply a zooming technique Klypin et al. 2001) to select a lagrangian volume of 3 virial radius centered in a MW-size halo at redshift 0 . Then, we resimulate that volume 


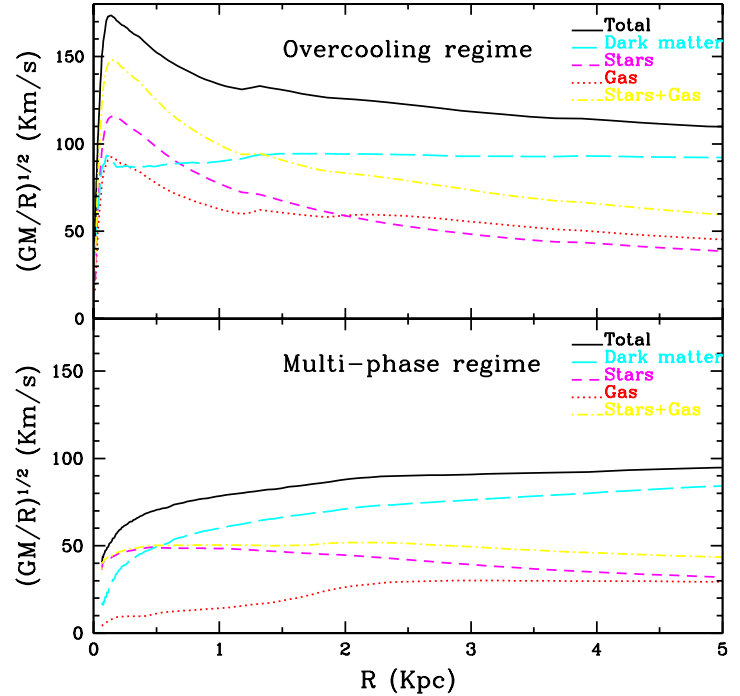

Fig. 12.- Circular velocity profile of the main progenitor of a MW-type galaxy at redshift 5 . The top panel shows the results of an inefficient stellar feedback. The galaxy is too concentrated and has a too massive spheroidal component. By contrast, the bottom panel shows a regime in which stellar feedback is more efficient and it can regulate the growth of the galaxy. The maximum resolution in both cases is $70 \mathrm{pc}$.

with higher resolution. The region has a radius of about $1.5 h^{-1}$ comoving Mpc. The simulation has about 5 million dark matter particles. They have three different masses. The high-resolution region is resolved with 3.4 million dark matter particles with a $7.5 \times 10^{5} \mathrm{M}_{\odot}$ mass per particle. The high-resolution volume is resolved with about 17 million volume elements at different levels of resolution. The maximum resolution is always between 35 and 70 pc. A short summary of the details of the simulations is given in table 1. The cosmological model assumed throughout the paper has $\Omega_{m}=0.3, \Omega_{\Lambda}=0.7, \mathrm{~h}=0.7$ and $\sigma_{8}=0.9$.

\subsection{Heating regime versus overcooling regime}

We compare two cosmological simulations with the same spatial resolution but different regimes. Table 1 shows a summary of the two simulations. The over-cooling model has low star formation ef-

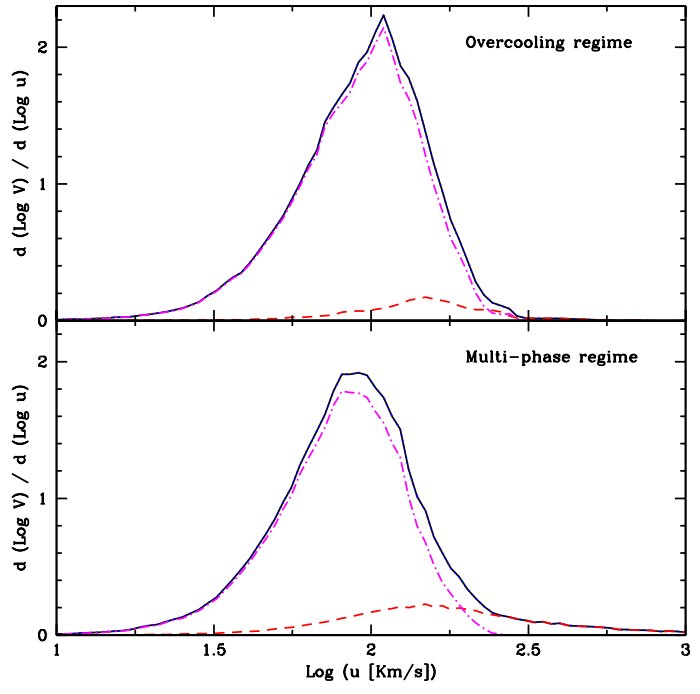

Fig. 13.- Distribution of velocities at the highest levels of the resolution for two models. The dashed curve represents the hot phase with $T>10^{4} \mathrm{~K}$ and the dash-dotted curve represents gas with temperatures bellow $10^{4} \mathrm{~K}$. The bottom panel shows a longer tail of high-velocities. These are galactic outflows which can reach velocities exceeding $10^{3}$ $\mathrm{km} \mathrm{s}^{-1}$.

ficiency. In addition, the cosmological UV background according to Haardt \& Madau (1996) is present over the whole evolution. Finally, a constant model of stellar feedback is used.

In the second simulation the heating regime develops. It has a high efficiency of star formation and the UV background is limited to its value at redshift 8 . The supernova model of stellar feedback was used in this case. We use a model of star formation in which each star formation event was treated as a random process (see Appendix). In this way, we keep a moderate galactic star formation rate of $\sim 10 \mathrm{M}_{\odot} \mathrm{yr}^{-1}$ inside the main galaxy at redshift 3 .

The simulation in the cooling regime has a cold galactic ISM with temperature close to $10^{4} \mathrm{~K}$. The simulation in the heating regime develops a 3 phase ISM. Hot bubbles develop naturally. They produce galactic chimneys that combine in a galactic wind. As a result, galactic winds are the natural outcome from stellar feedback. 
TABLE 1

PARAMETERS OF COSMOLOGICAL MODELS.

\begin{tabular}{lcc}
\hline \multicolumn{1}{c}{ Parameter } & Models \\
\hline Comoving box size & & $14.28 \mathrm{Mpc}$ \\
Number of DM particles & $5.4 \times 10^{6}$ \\
DM particle mass & $7.5 \times 10^{5} \mathrm{M}_{\odot}$ \\
Number of cells & $17.5 \times 10^{6}$ \\
Max. resolution (proper) & $35-70 \mathrm{pc}$ \\
Max. number of stars & & $3.7 \times 10^{6}$ \\
Min. mass of stellar particle & & $10^{4} \mathrm{M}_{\odot}$ \\
Model name & Overcooling & Multi-phase \\
UV flux & H\& M96 & H\& M96 but constant after $z=8$ \\
Star formation time scale $\tau$ & $4 \times 10^{7}$ yrs & $4 \times 10^{6}$ yrs \\
Model for stellar energy release & Constant & SN model \\
Runaway stars & not included & included \\
\hline
\end{tabular}

Figure 11 shows the main MW progenitor at redshift 3 for both simulations. The cooling model has a smooth density distribution with a small enhancement due to a pattern of spiral arms. In contrast, the multi-phase model develops a clumpy medium of dense clouds surrounded by low-density bubbles. This is a multi-phase medium.

\subsection{Comparison of circular velocity pro- files}

We can see now the effect of this multiphase medium in the galaxy assembly. We use the profile of circular velocity as a proxy of the mass distribution, $V_{c}=\sqrt{G M / R}$, where $G$ is the gravitational constant and $M$ is the mass inside a radius $R$. Figure 12 shows the profile of the circular velocity for the same galaxy in the two cases. The simulation with the overcooling problem shows a strong peak in the baryonic component of the circular velocity. Both gas and stars are very concentrated in the first Kpc. In contrast, the simulation with multiphase medium has a more shallow circular velocity profile. This indicates a less concentrated galaxy with less baryons. At the virial radius, $\mathrm{R}_{\text {vir }}=16 \mathrm{Kpc}$, the virial mass at $\mathrm{z}=5$ is 3.1 $\times 10^{10} \mathrm{M}_{\odot}$. A large fraction of this mass is dark matter, $\mathrm{M}_{d m}=2.7 \times 10^{10} \mathrm{M}_{\odot}$. The mass in gas is $\mathrm{M}_{\text {gas }}=0.24 \times 10^{10} \mathrm{M}_{\odot}$ and the baryons locked into stars accounts for only $0.14 \times 10^{10} \mathrm{M}_{\odot}$.

\subsection{Galactic winds and multiphase medium}

The hot bubbles in the multi-phase medium develop galactic fountains that produce hot outflows with very high velocities: larger than $10^{3} \mathrm{~km} \mathrm{~s}^{-1}$. These outflows are not produced in the cooling model. The Figure 13 shows the difference in the distribution function of velocities for both cases. We take all cells at the highest levels of refinement. Therefore, we select a volume close to the galaxies in the simulations. The multi-phase model has a bigger fraction of hot gas with much larger velocities than in the cooling model. These outflows contribute to the high-velocity tail of the distribution. In the cooling model, the distribution drops at $300 \mathrm{~km} \mathrm{~s}^{-1}$, while in the hot case the tail extends beyond $10^{3} \mathrm{~km} \mathrm{~s}^{-1}$.

These galactic-scale outflows can be seen figure 14. It shows a slice of the simulation through the main MW-progenitor at redshift $z=3.4$. At that redshift, its virial radius is $70 \mathrm{Kpc}$ and the total virial mass is $10^{11} \mathrm{M}_{\odot}$. The gas density panel shows the galaxy embedded in a cosmological web of filaments. The galaxy at the center is blowing a galactic wind of hot and dilute gas with outflows velocities exceeding $300 \mathrm{~km} \mathrm{~s}^{-1}$. The wind is rich in $\alpha$-elements and other products of the ejecta of core-collapse supernova. These metal-rich outflows can contribute to the enrichment of the halo and the inter-galactic medium. These outflows can reach even higher velocities and can escape the 


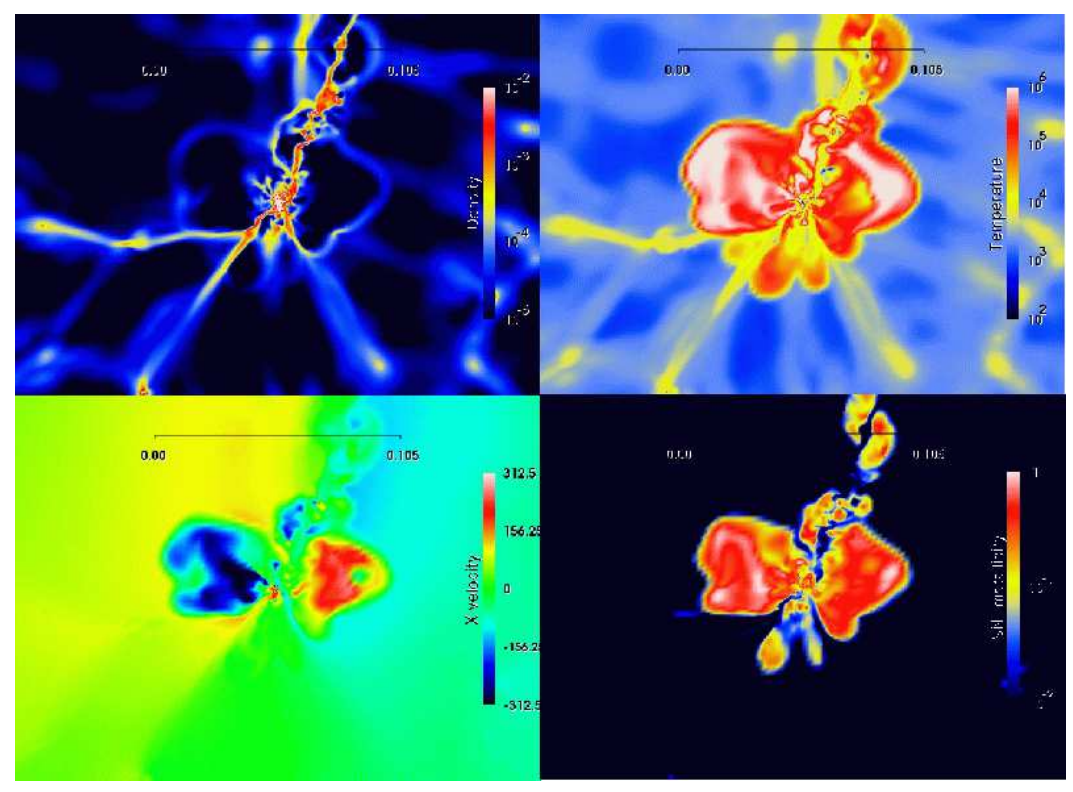

Fig. 14.- This panel shows a galaxy at redshift 3.4 with a resolution of $45 \mathrm{pc}$. The figures show slices of $600 \mathrm{Kpc}$ on a side of gas density (top left), temperature (top right), velocity in the horizontal direction (bottom left), and metallicity (bottom right). There are inflows of low-metallicity gas in cold filaments, as well as outflows of hot, metal-rich gas produced by chimneys in a multi-phase interstellar medium. Outflow velocities exceeds $300 \mathrm{~km} \mathrm{~s}^{-1}$. The virial radius is $70 \mathrm{Kpc}$ and the total virial mass is $10^{11}$ solar masses.

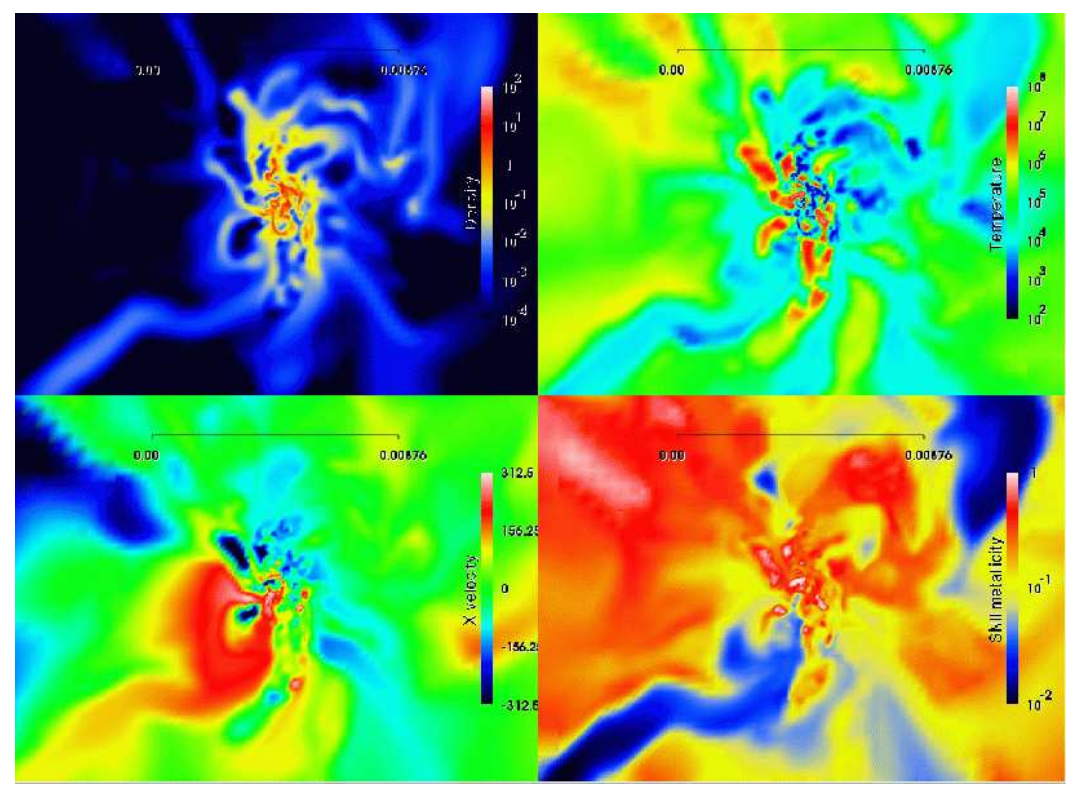

Fig. 15.- The same as in figure 14 but now the size of the images is $50 \mathrm{Kpc}$. It shows a multi-phase ISM of cold and dense clouds surrounded by bubbles of hot and dilute gas. Inflow and outflows velocities can reach $300 \mathrm{~km} \mathrm{~s}^{-1}$. The outflows are galactic chimneys powered by core-collapse supernova. Therefore, they are rich in $\alpha$-elements. In contrast, the inflow of gas has almost primordial composition. 


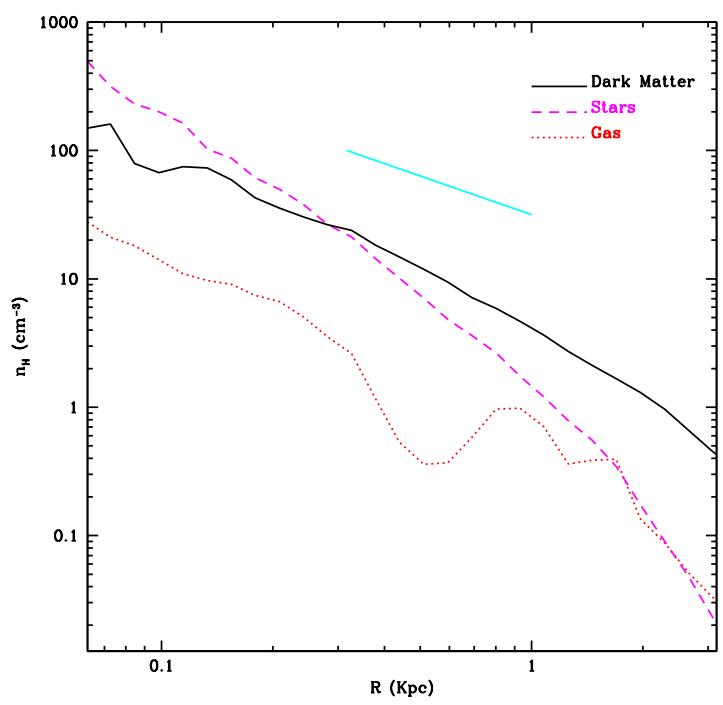

Fig. 16.- Density profile of a galactic halo at redshift 5 . The dark matter distribution is consistent with a cusp. The diagonal line shows a slope of -1 .

galactic halo and enrich the inter-galactic medium. The galactic wind is produced by the combination of different galactic chimneys anchored in the multi-phase ISM of the galaxy.

Figure [15] shows this multi-phase ISM. Cold and dense clouds coexist with low-density bubbles filled with very hot gas. Warm gas with intermediate densities and temperatures filled areas of low star formation and inflows of gas with almost primordial composition.

\subsection{Density profile consistent with a cuspy profile}

Figure 16 shows the inner profile of density of the different components of the galaxy: dark matter, gas and stars at redshift 5. The density slope of the dark matter profile is consistent with a cuspy profile. In contrast, Mashchenko et al. (2007) reported the formation of a core rather than a cusp in the central $\sim 300 \mathrm{pc}$ of a much smaller galaxy at high redshift $\left(\sim 10^{9} \mathrm{M}_{\odot}\right.$ at $\left.\mathrm{z}=6\right)$ in a $\mathrm{SPH}$ cosmological simulation. In their case, the mechanism that removes the cusp is gravitational heating from large fluctuations in the gravitational field. These fluctuations are produced by bulk motions of gas clumps driven by stellar feedback
(Mashchenko et al. 2006). These motions remove episodically $90 \%$ of the mass from the central 100 pc after each burst of star formation.

However, these gas clumps can be overproduced in simulations if the local Jeans length is not resolved (Truelove et al. 1997). This produces an artificial gas fragmentation and big clumps of stars. An excessive clumpiness can artificially increase the efficiency of this gravitational heating. In our simulations, we prevent this artificial fragmentation by the implementation of a pressure floor that increases the effective Jeans length to the resolution limit (Robertson \& Kravtsov 2007). However, a direct comparison between our results and Mashchenko et al. (2007) is difficult because we follow the formation of a much bigger galaxy $(\sim$ $10^{10} \mathrm{M}_{\odot}$ at $\mathrm{z}=5$ ), in which the effect of this gravitational heating driven by stellar feedback is less important. Therefore, these gravitational heating driven by stellar feedback can not be ruled out in low-mass and gas-rich starburst galaxies.

\section{Summary and conclusions}

We study the role of supernova explosions and stellar winds in the formation of galaxies. Our approach is to model these processes without the adhoc assumptions typically used on stellar feedback. Unlike many currently used prescriptions, we do not stop cooling in regions where the energy from stellar feedback is released (Thacker \& Couchman 2000; Brook et al. 2004; Keres et al. 2005; Governato et al. 2007). Moreover, instead of using a sub-resolution model of a multi-phase medium ( Springel \& Hernquist 2003, Cox et al. 2006), we resolve that multi-phase medium. This is a more straightforward way to model stellar feedback. It eliminates many ad-hoc assumptions. This approach also produces naturally the outcomes usually associated to stellar feedback: hot bubbles, chimneys and galactic winds.

Feedback heating has an effect in the ISM only when it dominates over radiative cooling. Section 2 shows the necessary conditions for this heating regime (eq. (5.77)). We find that a model of cooling bellow $10^{4} \mathrm{~K}$ is a key ingredient to fulfill these conditions. Thus, by resolving the conditions of molecular clouds $\left(T \approx 100 \mathrm{~K}\right.$ and $\left.n_{H}>10 \mathrm{~cm}^{-3}\right)$, we resolve the conditions, in which stellar feedback is more efficient in the ISM on galactic scales. 
We perform parsec-resolution simulations of a piece of a galactic disk in order to see the effects of stellar feedback and to test our models. When we use a realistic feedback and high resolution, the system has a low star formation rate and it forms hot super-bubbles of 100-pc scales and Kpcscale galactic chimneys. We found that the cores of these chimneys reach temperatures of $10^{7}-10^{8}$ $\mathrm{K}$, very low densities $\left(n_{H}<10^{-4} \mathrm{~cm}^{-3}\right)$, and outflow velocities exceeding $10^{3} \mathrm{~km} \mathrm{~s}^{-1}$.

Then, we degrade the resolution to see if this picture of multi-phase ISM holds at a resolution that we can achieve in cosmological simulations. We found that runaway stars help to spread the effect of stellar feedback. They usually explode as supernovae in low-density regions, few 100 pc away from their natal molecular cloud. This is an effect found in nature (Stone 1991), which enhances the feedback. So, it should be included in any realistic model of stellar feedback.

Thermal feedback from young stars is able to produce long time-scales of gas consumption by dissipating the star-forming gas. As a result, although this gas has high star formation efficiency, subsequent feedback processes produce a low star formation rate, averaged over all cold and dense gas. For example, in the simulations of the ISM described in $\S 4$, the gas with a density above the density threshold for star formation can form stars with high efficiency. However, the average star formation efficiency in the simulated clouds is roughly $2.5 \%$ over a freefall time-scale (\$4.3). This is roughly consistent with estimations of the star formation efficiency in molecular clouds (Zuckerman \& Evans 1974; Krumholz \& McKee 2005; Krumholz \& Tan 2007).

In cosmological simulations ( 55$)$, We find a moderate galaxy star formation rate, $\mathrm{SFR}=10$ $\mathrm{M}_{\odot} \mathrm{yr}^{-1}$ and a significant amount of cold and dense star-forming gas, $M_{\text {dense gas }}=10^{9} \mathrm{M}_{\odot}$ inside a $5 \mathrm{Kpc}$ star-forming disk at redshift 3 . These values are consistent with observations of nearby starburst galaxies. Using the observed relation between the star formation rate and the amount of star-forming gas of Gao \& Solomon (2004), the star formation rate expected for $10^{9} \mathrm{M}_{\odot}$ of cold and dense gas is $20 \mathrm{M}_{\odot} \mathrm{yr}^{-1}$. This is close to the value found in your simulations. Moreover, the galactic gas consumption time-scale of dense gas,
$\mathrm{M}_{\text {dense gas }} / \mathrm{SFR}$ is $\sim 100 \mathrm{Myr}$. This is consistent with observed values in local starburst galaxies which are usually used as analogs of star-forming galaxies at high redshift (Kennicutt 1998).

In our simulations, star formation proceeds in a way consistent with observations of star-forming galaxies (Kennicutt 1998). From the numbers given above, the gas surface density of the starforming disk of $5 \mathrm{Kpc}$ radius at redshift 3 is $\Sigma_{\text {gas }}=13 \mathrm{M}_{\odot} \quad \mathrm{pc}^{-2}$. Using the Kennicutt fit for nearby star-forming galaxies (Kennicutt 1998), the expected value for the star formation rate surface density is $\Sigma_{\mathrm{SFR}}=10^{-2} \mathrm{M}_{\odot} \mathrm{yr}^{-1} \mathrm{Kpc}^{-2}$. The measured value from the simulations is $\Sigma_{\mathrm{SFR}}=$ $1.3 \times 10^{-1} \mathrm{M}_{\odot} \mathrm{yr}^{-1} \mathrm{Kpc}^{-2}$. Although this value is an order of magnitude higher than the expected value from the fit, it is still within the intrinsic spread found in observations. As a result, our simulated high-redshift galaxy seems more compact than the average star-forming galaxy at lowredshift.

Our cosmological simulations with this model of stellar feedback do not have the overcooling problem. The fraction of cold baryons (stars and gas with a temperature bellow $10^{4} \mathrm{~K}$ ) inside the virial radius at $\mathrm{z}=5$ is 0.6 times the cosmological value ( $\left.f_{\text {cosmo }}=0.15\right)$. This is consistent with galaxy mass models (Klypin et al. 2002). Instead of a cold disk, we produce a multi-phase ISM with the same features seen in the simulations of the ISM described in section 4: cold clouds, hot super-bubbles and galactic chimneys. The angular momentum problem is also reduced. Instead of a compact object with a strong peak in the rotation curve, we produce more extended galaxies with nearly flat rotation curves. Baryons are less concentrated when stellar feedback plays a role in the formation of galaxies. At the same time, the density profile of dark matter is still consistent with a cuspy profile.

In this picture, galactic chimneys powered by stellar feedback combine into a galactic wind. So, galactic winds appear as the natural outcome of stellar feedback in starburst galaxies at high redshifts. We found typical outflow velocities of 300 $\mathrm{km} \mathrm{s}^{-1}$ with some exceptional examples of outflows exceeding $1000-2000 \mathrm{~km} \mathrm{~s}^{-1}$. This is consistent with observation of outflows at high redshift (Law et al. 2007). From a sample of $\approx 100$ galaxies at redshift $1.9<z<2.6$, Steidel et al. (2007) find a mean outflow velocity of $445 \mathrm{~km} \mathrm{~s}^{-1}$. Some 
cases have velocities of $1000 \mathrm{~km} \mathrm{~s}^{-1}$.

This picture is only reproduced if the resolution is high enough to resolve the physical conditions of densities and temperatures of molecular clouds. Our cosmological simulations reach a resolution of $35 \mathrm{pc}$, which is 10 times better than the typical resolution in previous cosmological simulations (Sommer-Larsen et al. 2003; Abadi et al. 2003; Robertson et al. 2004; Brook et al. 2004; Okamoto et al. 2005; Governato et al. 2007).

\section{Acknowledgments}

We are grateful to A. Kravtsov for providing the hydro code. We are in debt to N. Gnedin for giving us the wonderful analysis and graphics package IFRIT. We thank K. Tassis for very useful discussions. We acknowledge support of NSF grants to NMSU. The computer simulations presented in this paper were performed at the National Energy Research Scientific Computing Center (NERSC) of the Lawrence Berkeley National Laboratory and at the NASA Advanced Supercomputing (NAS) Division of NASA Ames Research Center. 


\section{A. A model of star formation for scales bellow $100 \mathrm{pc}$}

A successful model of star formation in simulations should take into account the spatial resolution. For example, in typical cosmological simulations with a resolution of $\sim 1 \mathrm{Kpc}$, the star formation is averaged over a large piece of ISM. These simulations should have a star formation model with a low star formation efficiency in order to reproduce the global efficiencies found in nearby galaxies. Observations of quiescent galactic disks show long gas consumption time-scales averaged over a significant piece of a galaxy, $\tau_{\text {global }}=\Sigma_{\text {gas }} / \Sigma_{\text {SFR }} \sim 1$ Gyr, where $\Sigma_{\mathrm{SFR}}$ is the star formation rate surface density an $\Sigma_{\text {gas }}$ is the gas surface density (Kennicutt 1998; Kennicutt et al. 2007). At the same time, for starburst galaxies, the global gas consumption time-scale is much shorter, $\tau_{\text {global }}=0.1 \mathrm{Gyr}$ (Kennicutt 1998).

However, if the resolution is high enough to resolve the regions where star formation mainly occurs, giant molecular clouds, the star formation efficiency can be much higher: the time-scales for the formation of Galactic stellar clusters are around few Myr and 10\% - 40\% of the gas is consumed (Greene \& Young 1992; Elmegreen et al. 2000). As a result, simulations which can resolve the sites of star formation should have a high star formation efficiency only in the high-density regions, where molecular clouds can form (Tasker \& Brvan 2006). In practice, the maximum resolution that we can afford is between 30-70 pc. This limits the maximum density that our simulations can resolve. For example, if we consider a typical giant molecular cloud of $10^{5} \mathrm{M}_{\odot}$ (Rosolowsky et al. 2007), the mean density averaged over 30-80 pc scales will be $10-200 \mathrm{~cm}^{-3}$. This gives an idea of the typical densities where star formation occurs our simulations.

In our code, star formation is allowed in a time step, $d t_{\mathrm{SF}}$, which is equal to the time step of the 0Level of resolution. This time step is controlled by the Courant condition for hydrodynamics and in our cosmological simulations, $d t_{\mathrm{SF}}=1-2 \mathrm{Myr}$. During this period of time, a stellar particle can form only where the density and temperature reach a given threshold: $\rho_{\text {gas }}>\rho_{\mathrm{SF}}$ and $T_{\mathrm{gas}}<T_{\mathrm{SF}}$. Even in these cold and dense regions, each star formation event is treated as a random event with a probability $\operatorname{Pr}$ to occur. We roughly approximate the fact that regions with higher densities have a higher probability to host star formation events by assuming a simplified formula:

$$
\operatorname{Pr}=\frac{\rho_{\mathrm{gas}}}{100 \rho_{\mathrm{SF}}}
$$

In this way, the number of stellar particles remains in a value that is not computational prohibited. In the formation of a single stellar particle, the star formation rate is proportional to the gas density (Kravtsov 2003):

$$
\frac{d \rho_{*, \text { young }}}{d t}=\frac{\rho_{\text {gas }}}{\tau}
$$

where $\rho_{*, \text { young }}$ is the density of new stars, $\rho_{\text {gas }}$ is the gas density and $\tau$ is a constant star formation timescale. The density and temperature thresholds used are $\rho_{\mathrm{SF}}=0.035 \mathrm{M}_{\odot} \mathrm{pc}^{-3}\left(n_{H}=1 \mathrm{~cm}^{-3}\right)$ and $T_{\mathrm{SF}}=10^{4} \mathrm{~K}$. In spite of the fact that we allow star formation starting at $10^{4} \mathrm{~K}$, in practice the vast majority (>90\%) of "stars" form at temperatures below $1000 \mathrm{~K}$ and more than half of the stars form bellow $300 \mathrm{~K}$ and densities larger than $10 \mathrm{~cm}^{-3}$.

As described in $\S 2.1$, the ratio $\rho_{*, \text { young }} / \rho_{\text {gas }}$ should be $\sim 0.1-0.5$ for typical conditions of dense, starforming gas. Only in this case thermal feedback can produce over-pressured hot bubbles in the sites of star formation (eq. 5). Based on equation A2 this ratio of densities can be expressed as

$$
\frac{\rho_{* \text {,young }}}{\rho_{\text {gas }}}=\frac{d t_{\mathrm{SF}}}{\tau}
$$

As a result, thermal feedback is only efficient in dense, cold, star-forming gas if $d t_{\mathrm{SF}} / \tau \sim 0.1-0.5$. This sets the value of $\tau$, because $d t_{\mathrm{SF}}$ is set by the conditions of hydrodynamics, as explained before: $d t_{\mathrm{SF}}=1-2 \mathrm{Myr}$. Therefore, the value of $\tau$ should be in the range 2-20 Myr, consistent with the gas consumption time-scales during the formation of Galactic stellar clusters (Greene \& Young 1992; Elmegreen et al. 2000). However, 
this high local efficiency of star formation in high-density regions produces the observed low global efficiency, $\tau_{\text {global }}=0.1-1 \mathrm{Gyr}$, as discussed in $\S 4.3$. 


\section{REFERENCES}

Abadi, M. G., Navarro, J. F., Steinmetz, M., \& Eke, V. R. 2003, ApJ, 591, 499

de Avillez, M. A. 2000, MNRAS, 315, 479

de Avillez, M. A., \& Breitschwerdt, D. 2004, A\&A, 425,899

de Avillez, M. A., \& Breitschwerdt, D. 2007, ApJ, 665, L35

Blaauw, A. 1961, Bull. Astron. Inst. Netherlands, 15,265

Blumenthal, G. R., Faber, S. M., Primack, J. R., \& Rees, M. J. 1984, Nature, 311, 517

Brook, C. B., Kawata, D., Gibson, B. K., \& Freeman, K. C. 2004, ApJ, 612, 894

Clark, P. C., Bonnell, I. A., Zinnecker, H., \& Bate, M. R. 2005, MNRAS, 359, 809

Cox, D. P. 2005, ARA\&A, 43, 337

Cox, T. J., Jonsson, P., Primack, J. R., \& Somerville, R. S. 2006, MNRAS, 373, 1013

Davis, M., Efstathiou, G., Frenk, C. S., \& White, S. D. M. 1985, ApJ, 292, 371

Dekel, A., \& Silk, J. 1986, ApJ, 303, 39

D'Onghia, E., Burkert, A., Murante, G., \& Khochfar, S. 2006, MNRAS, 372, 1525

Dray, L. M., Dale, J. E., Beer, M. E., Napiwotzki, R., \& King, A. R. 2005, MNRAS, 364, 59

Elmegreen, B. G., Efremov, Y., Pudritz, R. E., \& Zinnecker, H. 2000, Protostars and Planets IV, 179

Feigelson, E. D., et al. 2005, ApJS, 160, 379

Ferland, G. J., Korista, K. T., Verner, D. A., Ferguson, J. W., Kingdon, J. B., \& Verner, E. M. 1998, PASP, 110, 761

Ferrière, K. 1998, ApJ, 503, 700

Ferrière, K. M. 2001, Reviews of Modern Physics, 73,1031

Gao, Y., \& Solomon, P. M. 2004, ApJ, 606, 271
Gerritsen, J. P. E., \& Icke, V. 1997, A\&A, 325, 972

Gies, D. R. 1987, ApJS, 64, 545

Governato, F., et al. 2004, ApJ, 607, 688

Governato, F., Willman, B., Mayer, L., Brooks, A., Stinson, G., Valenzuela, O., Wadsley, J., \& Quinn, T. 2007, MNRAS, 374, 1479

Greene, T. P., \& Young, E. T. 1992, ApJ, 395, 516

Guedel, M., Briggs, K. R., Montmerle, T., Audard, M., Rebull, L., \& Skinner, S. L. 2007, ArXiv e-prints, 712, arXiv:0712.0476

Haardt, F., \& Madau, P. 1996, ApJ, 461, 20

Hillenbrand, L. A., \& Hartmann, L. W. 1998, ApJ, 492,540

Hoogerwerf, R., de Bruijne, J. H. J., \& de Zeeuw, P. T. 2000, ApJ, 544, L133

Katz, N. 1992, ApJ, 391, 502

Kaufmann, T., Mayer, L., Wadsley, J., Stadel, J., \& Moore, B. 2006, MNRAS, 370, 1612

Kennicutt, R. C., Jr. 1998, ApJ, 498, 541

Kennicutt, R. C., Jr., et al. 2007, ApJ, 671, 333

Kereš, D., Katz, N., Weinberg, D. H., \& Davé, R. 2005, MNRAS, 363, 2

Klypin, A., Kravtsov, A. V., Bullock, J. S., \& Primack, J. R. 2001, ApJ, 554, 903

Klypin, A., Zhao, H., \& Somerville, R. S. 2002, ApJ, 573, 597

Kraichnan, R. H. 1967, Physics of Fluids, 10, 1417

Kravtsov, A. V., Klypin, A. A., \& Khokhlov, A. M. 1997, ApJS, 111, 73

Kravtsov, A. V. 1999, Ph.D. Thesis,

Kravtsov A., 2003, ApJ (Letters) 590, 1

Kroupa, P., Aarseth, S., \& Hurley, J. 2001, MNRAS, 321, 699

Krumholz, M. R., \& McKee, C. F. 2005, ApJ, 630, 250 
Krumholz, M. R., \& Tan, J. C. 2007, ApJ, 654, 304

Korpi, M. J., Brandenburg, A., Shukurov, A., Tuominen, I., \& Nordlund, Å. 1999, ApJ, 514, L99

Lada, C. J., \& Lada, E. A. 2003, ARA\&A, 41, 57

Law, D. R., Steidel, C. C., Erb, D. K., Larkin, J. E., Pettini, M., Shapley, A. E., \& Wright, S. A. 2007, ApJ, 669, 929

Leitherer, C., et al. 1999, ApJS, 123, 3

Mashchenko, S., Couchman, H. M. P., \& Wadsley, J. 2006, Nature, 442, 539

Mashchenko, S., Wadsley, J., \& Couchman, H. M. P. 2007, ArXiv e-prints, 711, arXiv:0711.4803

McCray, R., \& Snow, T. P., Jr. 1979, ARA\&A, 17, 213

McKee, C. F. 1999, NATO ASIC Proc. 540: The Origin of Stars and Planetary Systems, 29

Navarro, J. F., \& White, S. D. M. 1993, MNRAS, 265,271

Navarro, J. F., \& Steinmetz, M. 2000, ApJ, 538, 477

Norman, C. A., \& Ikeuchi, S. 1989, ApJ, 345, 372

Okamoto, T., Eke, V. R., Frenk, C. S., \& Jenkins, A. 2005, MNRAS, 363, 1299

Poveda, A., Ruiz, J., \& Allen, C. 1967, Boletin de los Observatorios Tonantzintla y Tacubaya, 4, 86

Robertson, B., Yoshida, N., Springel, V., \& Hernquist, L. 2004, ApJ, 606, 32

Robertson, B., \& Kravtsov, A. 2007, ArXiv eprints, 710, arXiv:0710.2102

Rosen, A., \& Bregman, J. N. 1995, ApJ, 440, 634

Rosolowsky, E., Keto, E., Matsushita, S., \& Willner, S. P. 2007, ApJ, 661, 830

Sheth, K., Vogel, S. N., Wilson, C. D., \& Dame, T. M. 2008, ApJ, 675, 330
Scalo, J., Vazquez-Semadeni, E., Chappell, D., \& Passot, T. 1998, ApJ, 504, 835

Slyz, A. D., Devriendt, J. E. G., Bryan, G., \& Silk, J. 2005, MNRAS, 356, 737

Sommer-Larsen, J., Götz, M., \& Portinari, L. 2003, ApJ, 596, 47

Spergel, D. N., et al. 2007, ApJS, 170, 377

Spitzer, L. J. 1990, ARA\&A, 28, 71

Springel, V., \& Hernquist, L. 2003, MNRAS, 339, 289

Steidel, C. C. et al., 2007, in preparation

Stone, R. C. 1991, AJ, 102, 333

Tasker, E. J., \& Bryan, G. L. 2006, ApJ, 641, 878

Thacker, R. J., \& Couchman, H. M. P. 2000, ApJ, 545,728

Townsley, L. K., Feigelson, E. D., Montmerle, T., Broos, P. S., Chu, Y.-H., \& Garmire, G. P. 2003, ApJ, 593, 874

Truelove, J. K., Klein, R. I., McKee, C. F., Holliman, J. H., II, Howell, L. H., \& Greenough, J. A. 1997, ApJ, 489, L179

Vázquez-Semadeni, E., Passot, T., \& Pouquet, A. 1995, ApJ, 441, 702

Vázquez-Semadeni, E., Ballesteros-Paredes, J., \& Klessen, R. S. 2003, ApJ, 585, L131

Wada, K., \& Norman, C. A. 2001, ApJ, 547, 172

Wada, K., \& Norman, C. A. 2007, ApJ, 660, 276

Wang, J., Townsley, L. K., Feigelson, E. D., Broos, P. S., Getman, K. V., Roman-Zuniga, C., \& Lada, E. 2007, ArXiv e-prints, 711, arXiv:0711.2024

Yepes, G., Kates, R., Khokhlov, A., \& Klypin, A. 1997, MNRAS, 284, 235

Zuckerman, B., \& Evans, N. J., II 1974, ApJ, 192, L149

Zwicky, F. 1957, Berlin: Springer, 1957,

This 2-column preprint was prepared with the AAS LATEX macros $\mathrm{v} 5.2$. 